Supporting information

\title{
PELE-MSM: a Monte Carlo based protocol for the estimation of absolute binding free energies
}

Joan F Gilabert ${ }^{1}$, Christoph Grebner ${ }^{4}$, Daniel Soler ${ }^{2}$, Daniel Lecina ${ }^{1}$, Marti Municoy ${ }^{1}$, Oriol Gracia Carmona ${ }^{1}$, Robert Soliva ${ }^{2}$, Martin J. Packer ${ }^{3}$, Samantha J. Hughes ${ }^{3}$, Christian Tyrchan ${ }^{4}$, Anders Hogner ${ }^{4}$ and Victor Guallar ${ }^{1,5}$

${ }^{1}$ Barcelona Supercomputing Center, Jordi Girona 29, E-08034 Barcelona, Spain

${ }^{2}$ Nostrum Biodiscovery, Jordi Girona 29, Nexus II D128, 08034, Barcelona, Spain ${ }^{3}$ Chemistry, R\&D Oncology, AstraZeneca, Cambridge CB4 OQA, United Kingdom ${ }^{4}$ Medicinal Chemistry, Research and Early Development Cardiovascular, Renal and Metabolism, BioPharmaceuticals R\&D, AstraZeneca, Gothenburg, Sweden ${ }^{5}$ ICREA, Passeig Lluís Companys 23, E-08010 Barcelona, Spain

2D structures of ligands used in the study

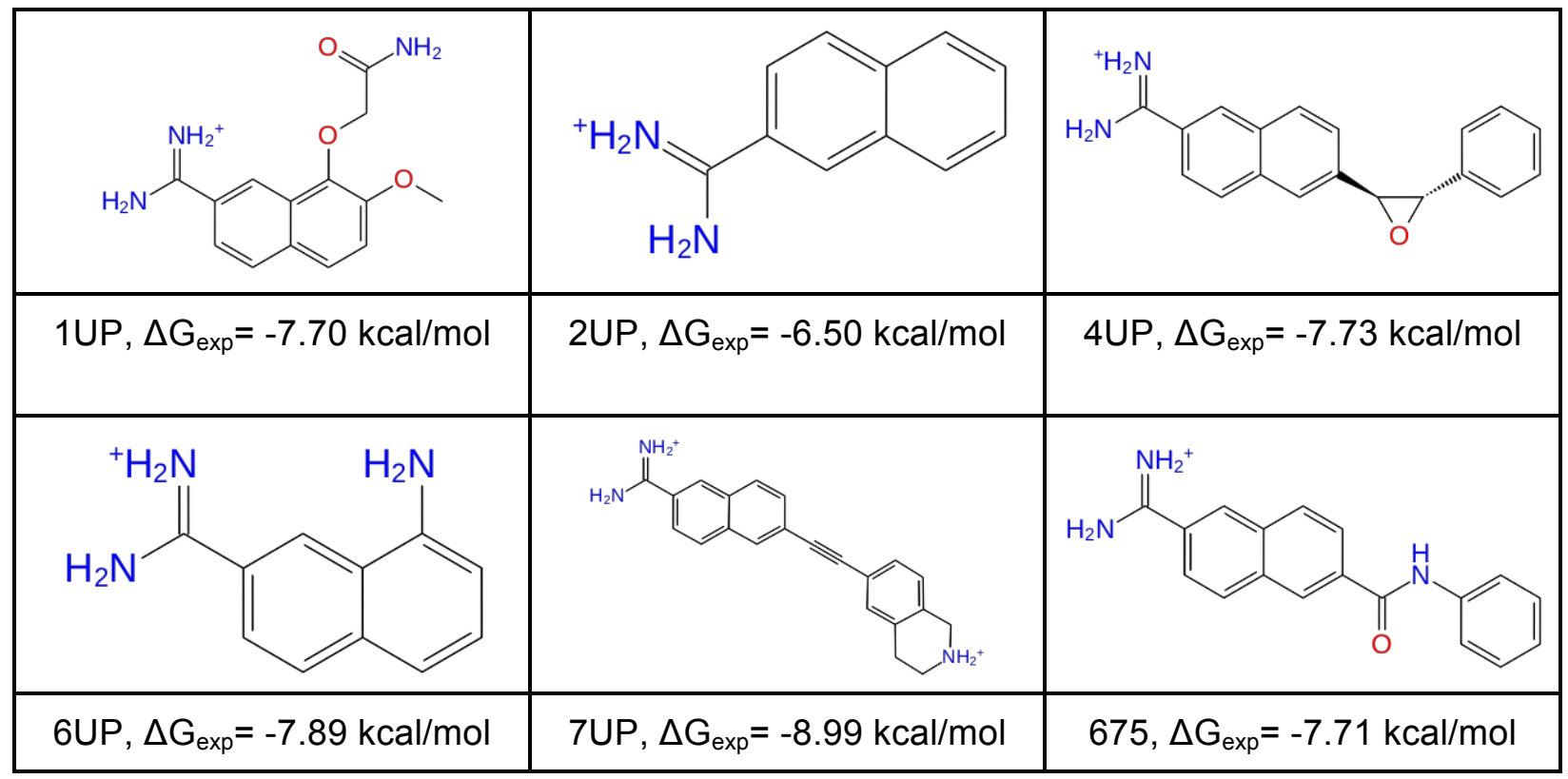


<smiles>NCc1ccc(NC(=O)c2ccc3cc(C(N)=[NH2+])ccc3c2)cc1</smiles>

239, $\Delta \mathrm{G}_{\mathrm{exp}}=-9.20 \mathrm{kcal} / \mathrm{mol}$

Table S1. Two dimensional structures of the ligands of the URO receptor, alongside with their experimental affinities

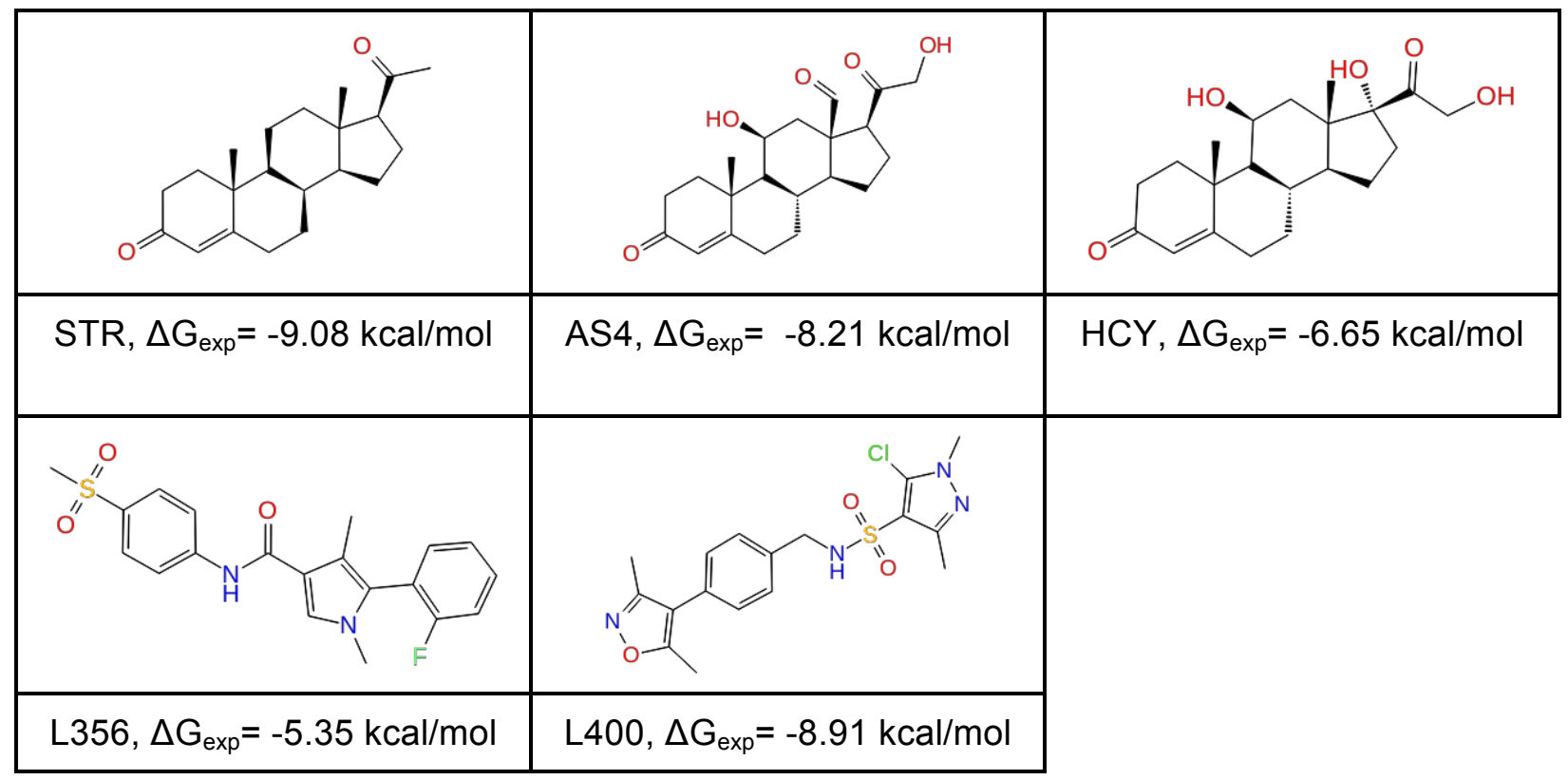

Table S2. Two dimensional structures of the ligands of the PR receptor, alongside with their experimental affinities

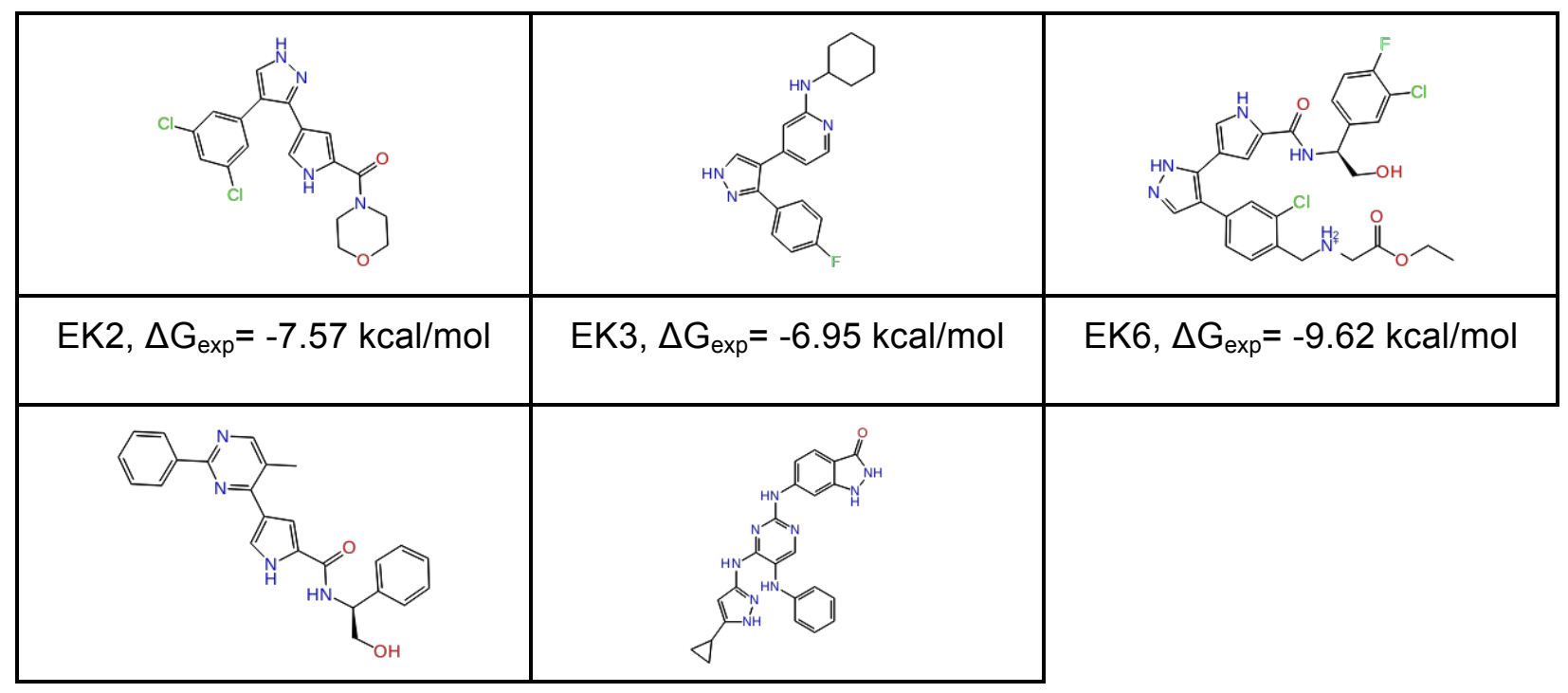


$\mathrm{EK} 9, \Delta \mathrm{G}_{\mathrm{exp}}=-9.62 \mathrm{kcal} / \mathrm{mol}$

$\mathrm{E} 63, \Delta \mathrm{G}_{\mathrm{exp}}=-9.72 \mathrm{kcal} / \mathrm{mol}$

Table S3. Two dimensional structures of the ligands of the ERK2 receptor, alongside with their experimental affinities

(n)

Table S4. Two dimensional structures of the ligands of the plasmi receptor, alongside with their experimental affinities

\section{Results for Plasmin system}
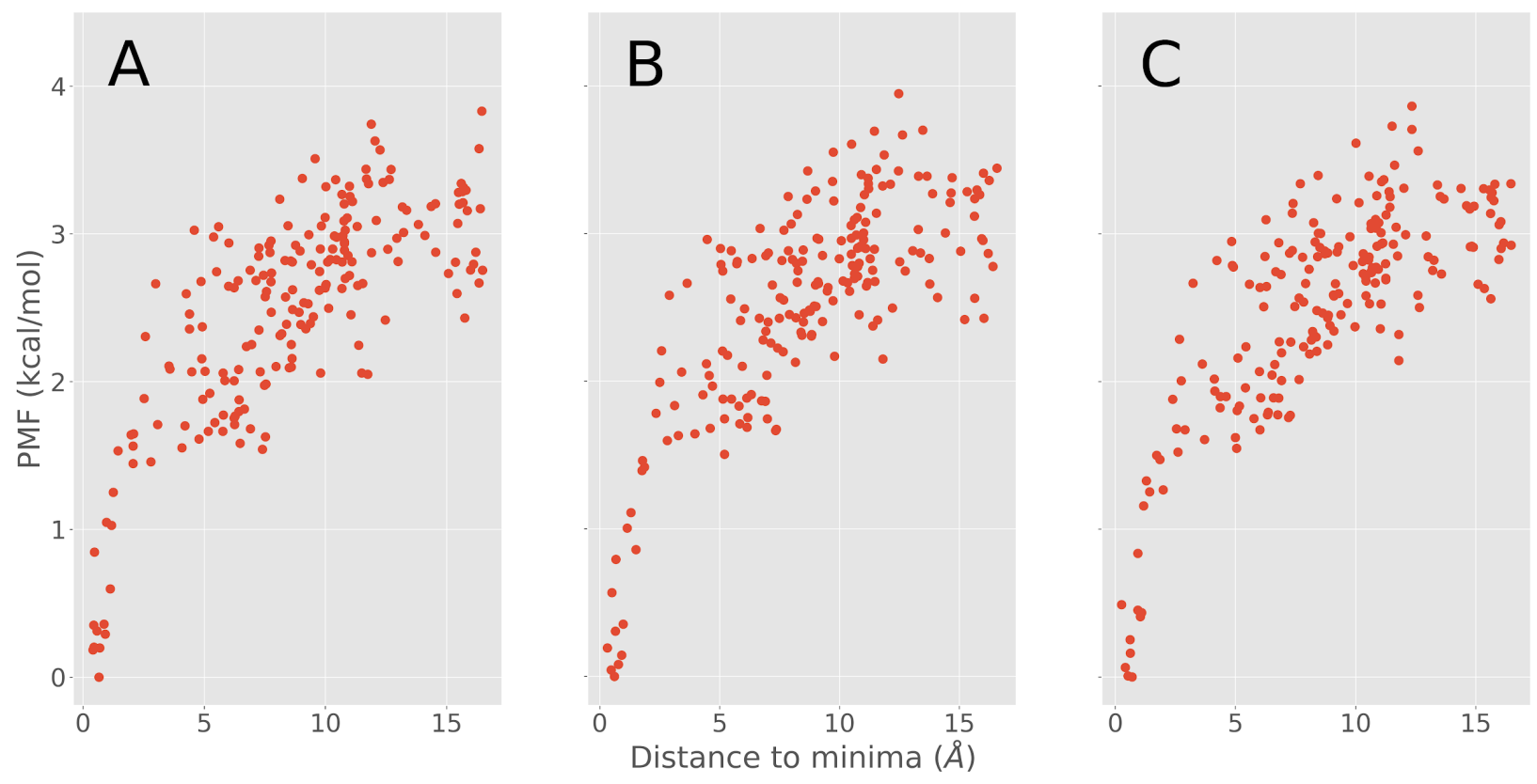
Figure S1. Comparison of the evolution of the one-dimensional PMF with three iterations for the L02 ligand of the plasmi receptor
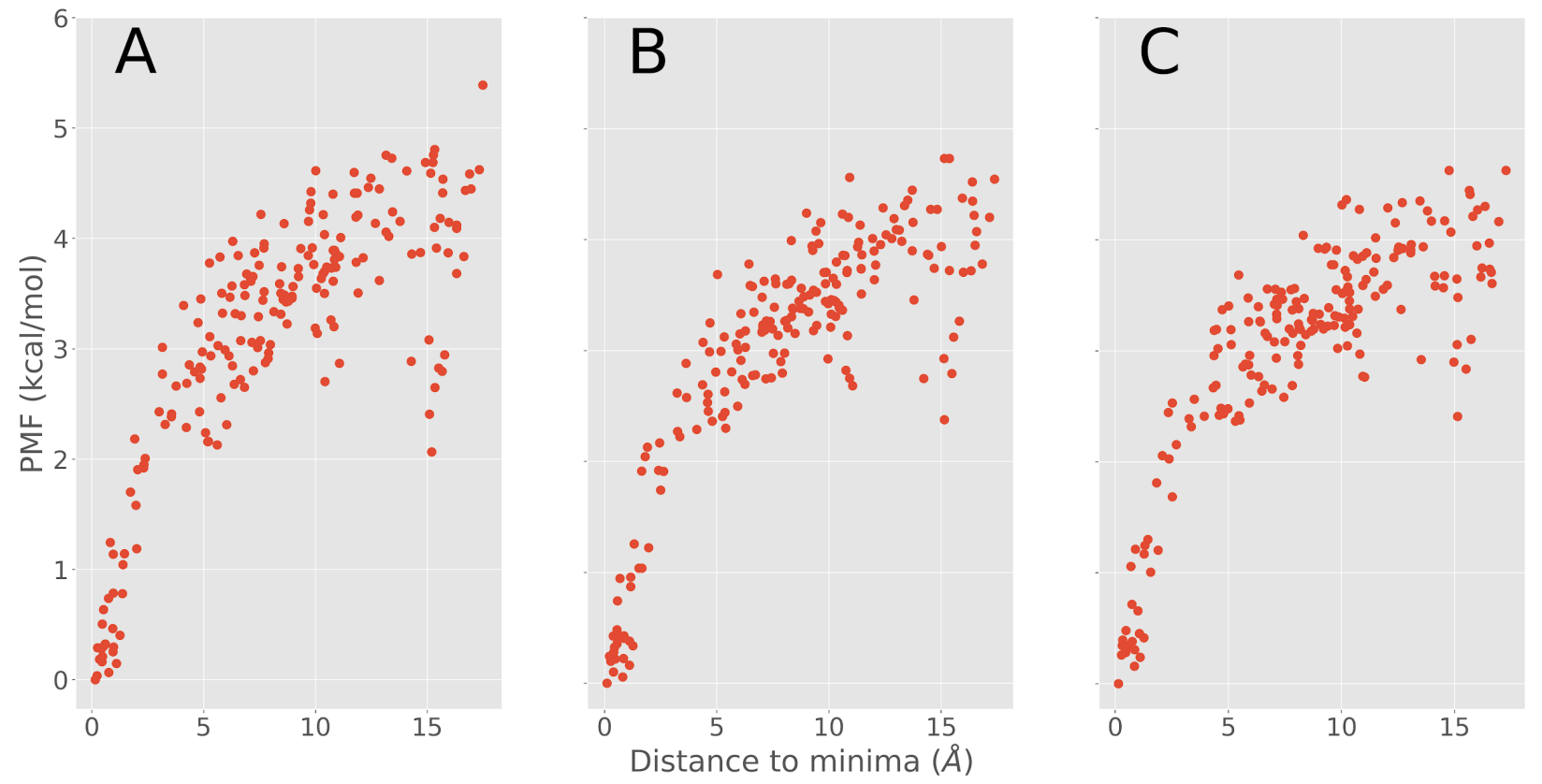

Figure S2. Comparison of the evolution of the one-dimensional PMF with three iterations for the L05 ligand of the plasmi receptor
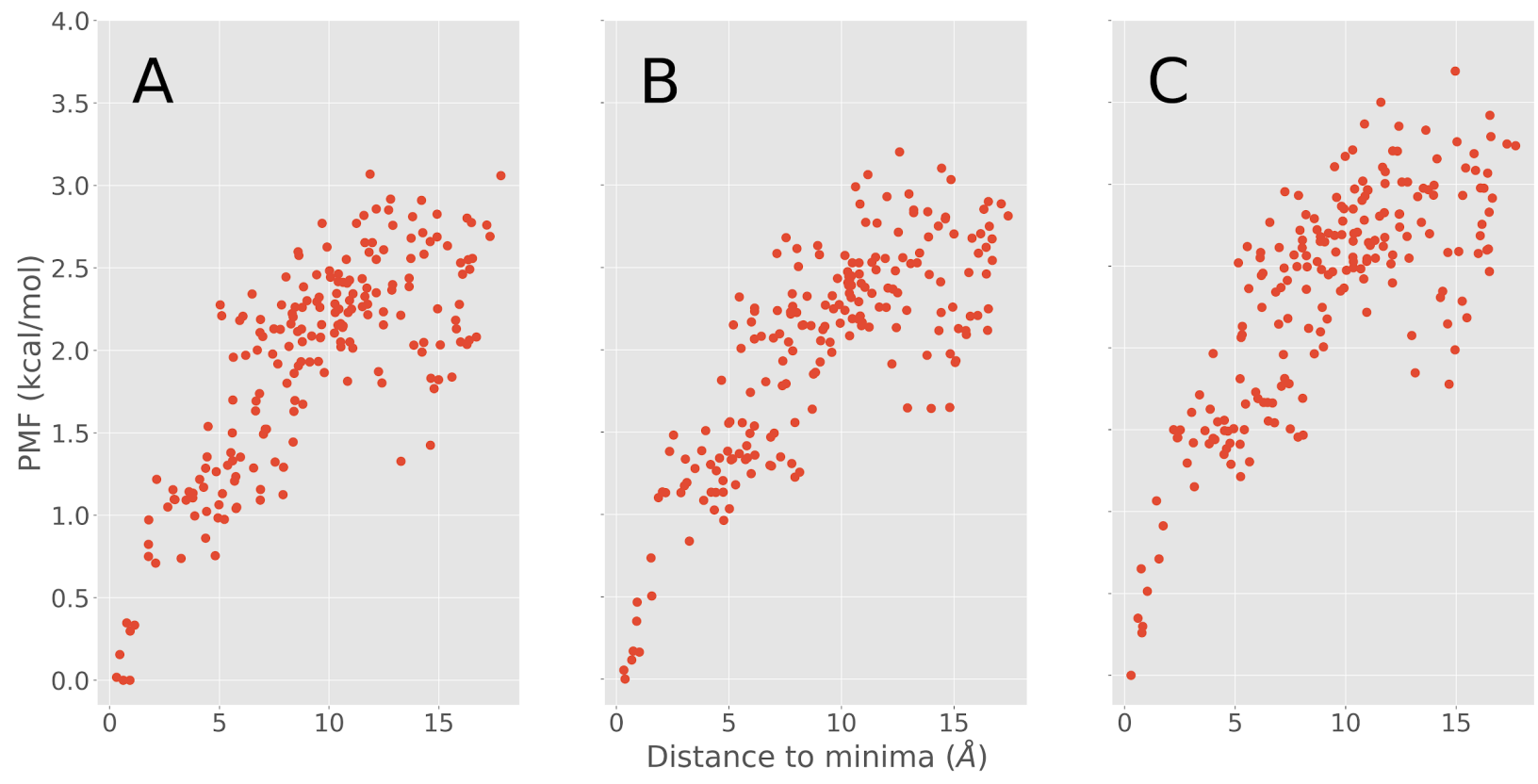

Figure S3. Comparison of the evolution of the one-dimensional PMF with three iterations for the L06 ligand of the plasmi receptor 

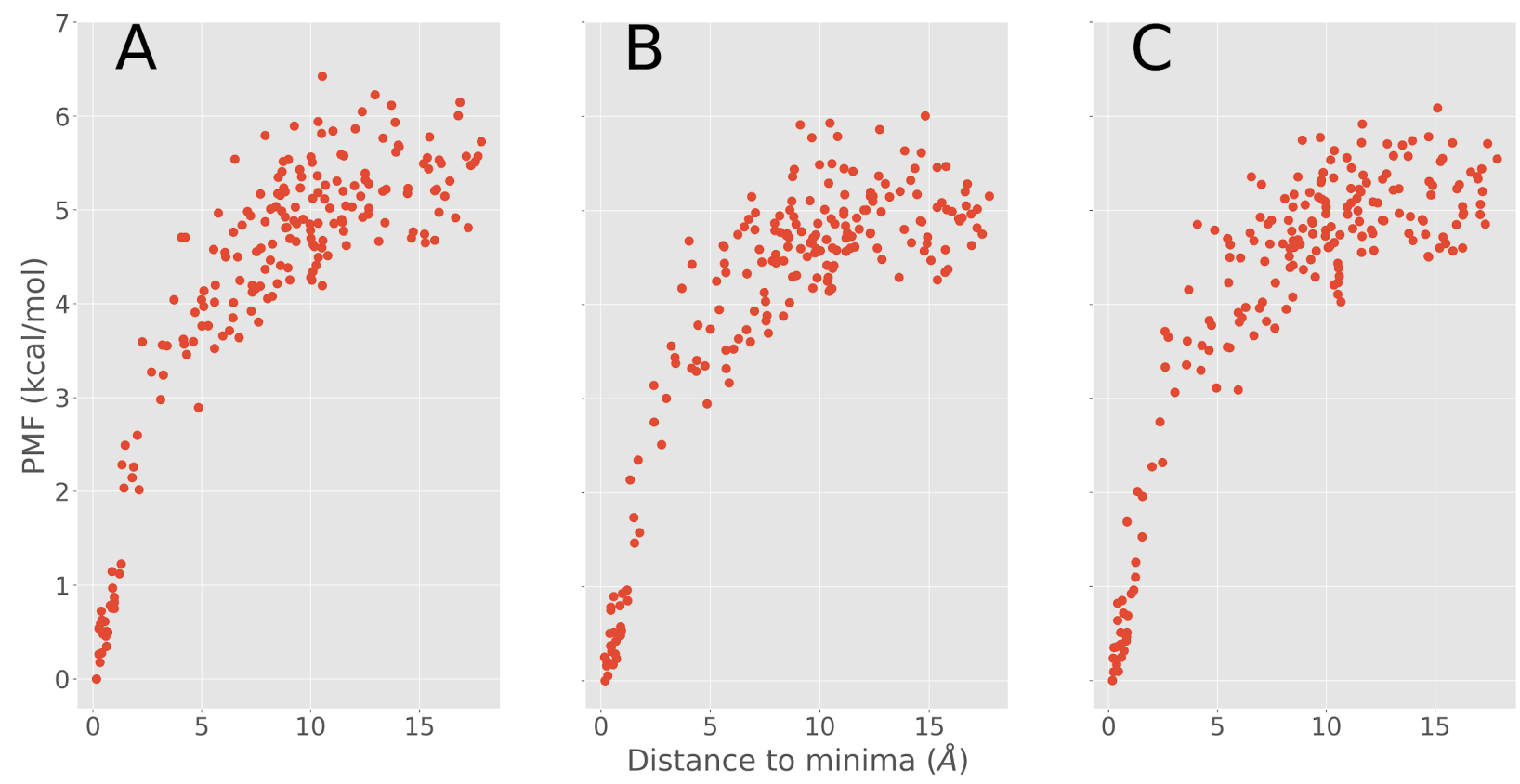

Figure S4. Comparison of the evolution of the one-dimensional PMF with three iterations for the L07 ligand of the plasmi receptor
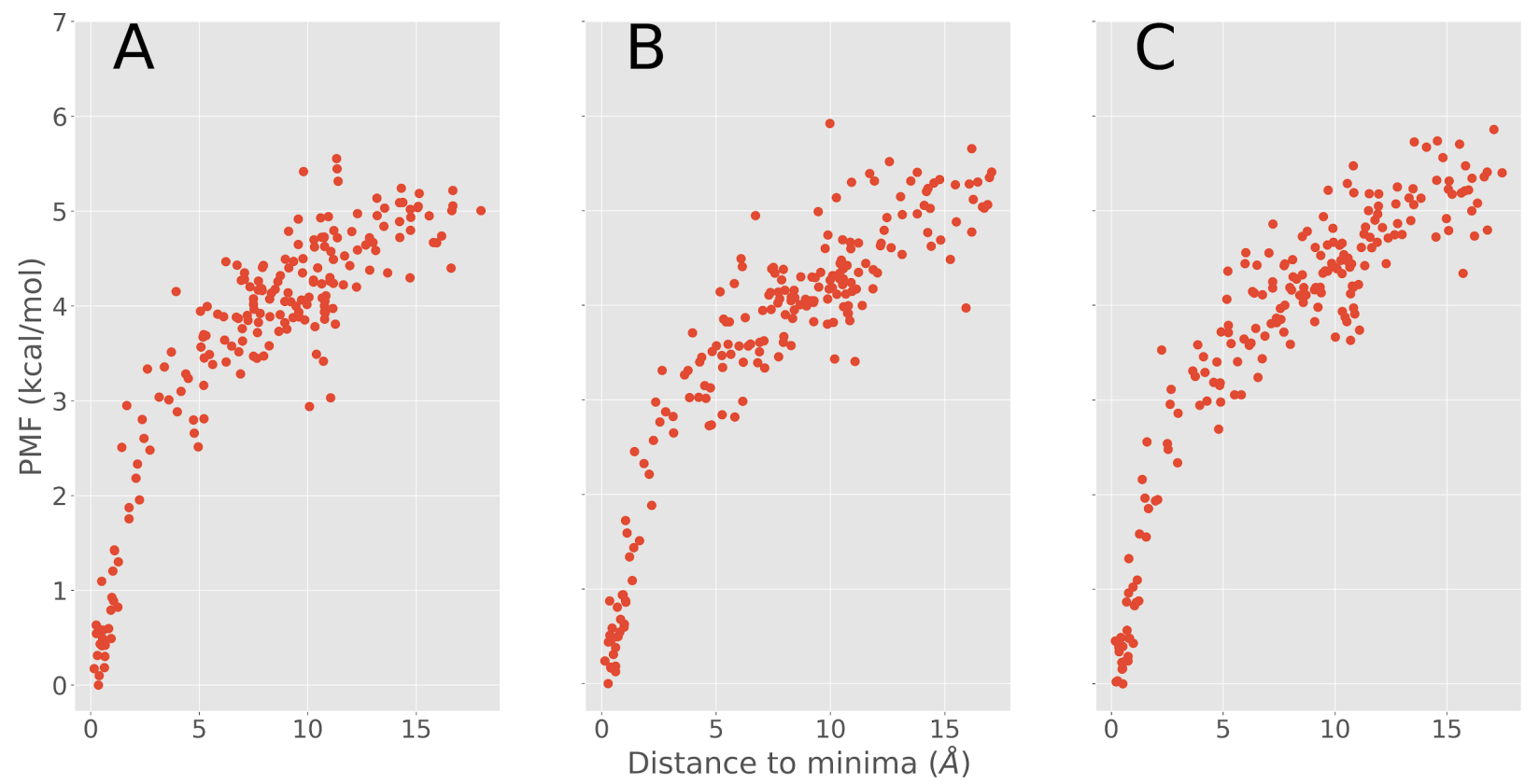

Figure S5. Comparison of the evolution of the one-dimensional PMF with three iterations for the L15 ligand of the plasmi receptor 

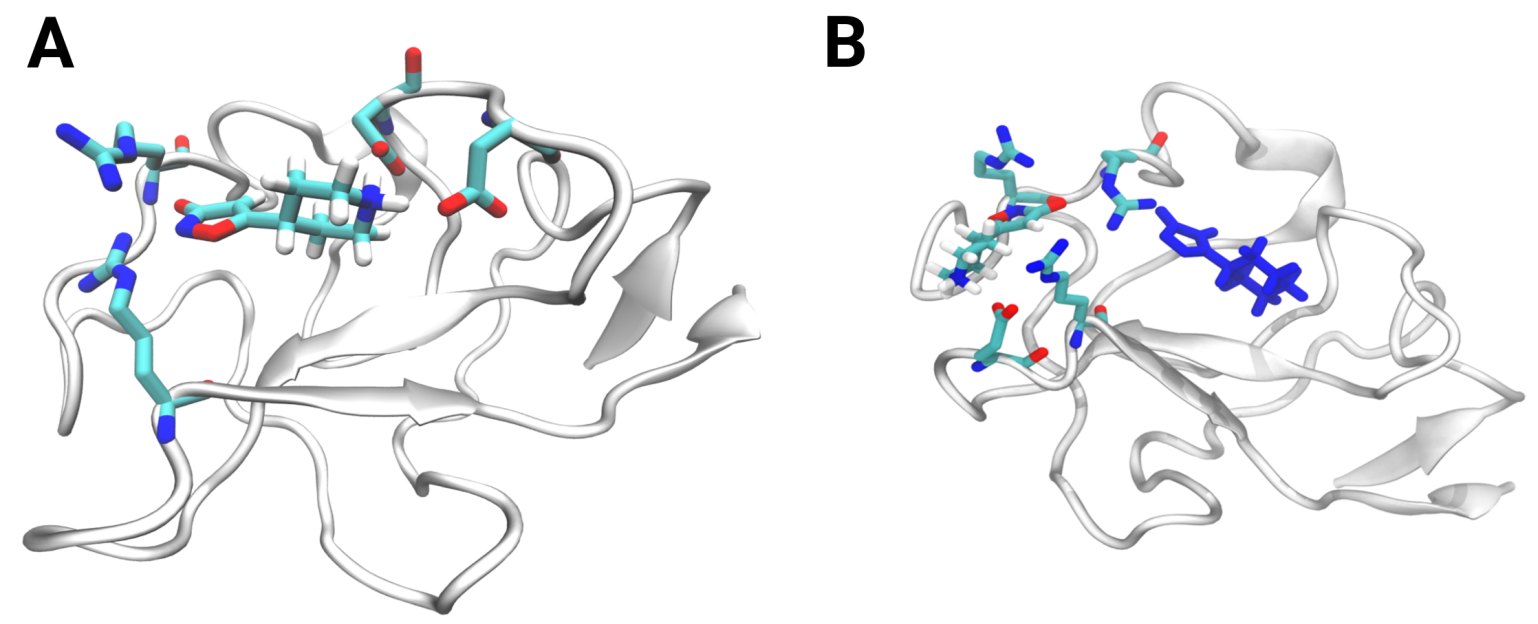

Figure S6. Representative structure of the minima of the L01 ligand (A) Active site and absolute minimum (B) Secondary minimum at about $10 \AA$ RMSD from the active site, with the active site structure depicted in blue for reference

\section{Results for URO system}
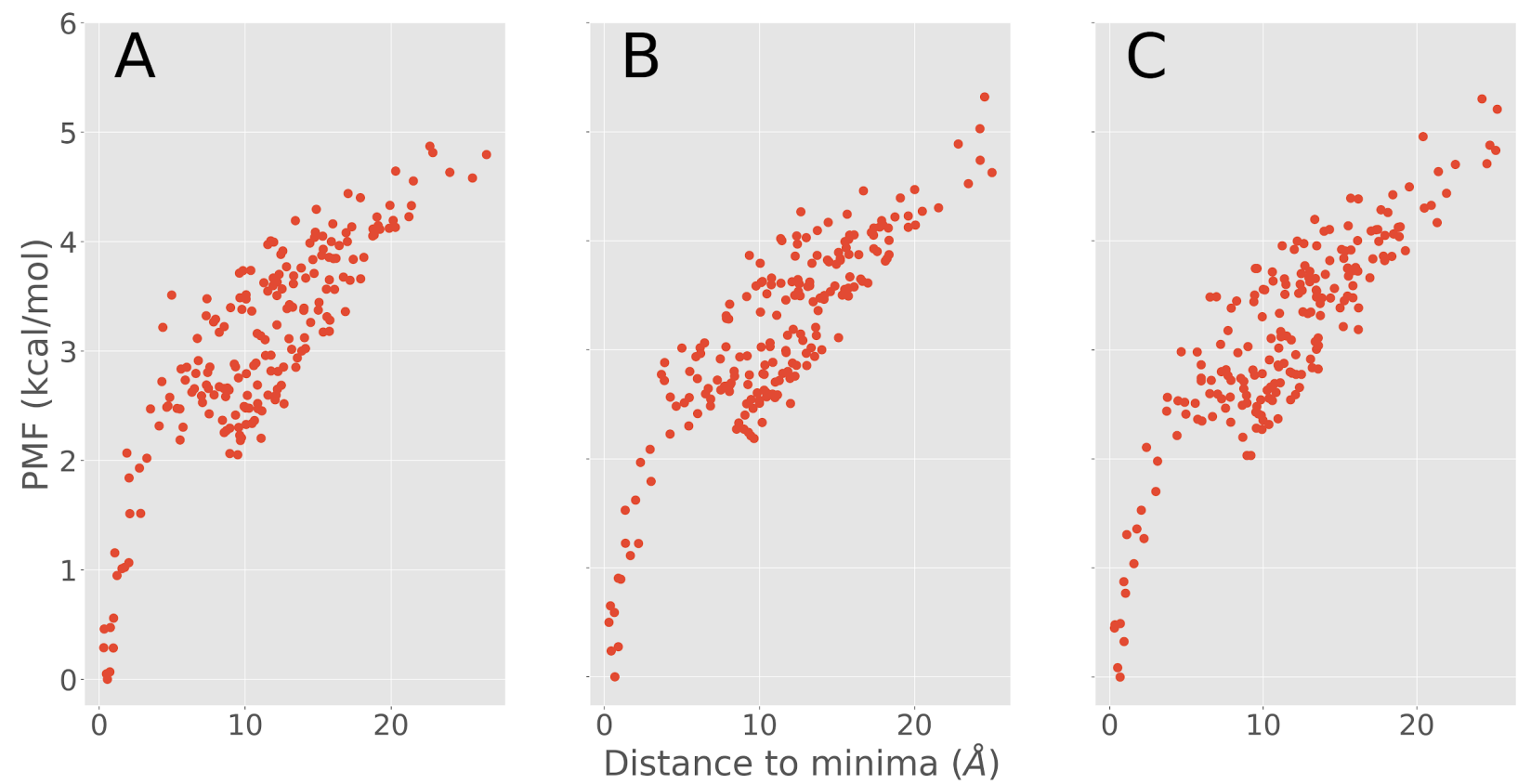

Figure S7. Comparison of the evolution of the one-dimensional PMF with three iterations for the 2UP ligand of the URO receptor 

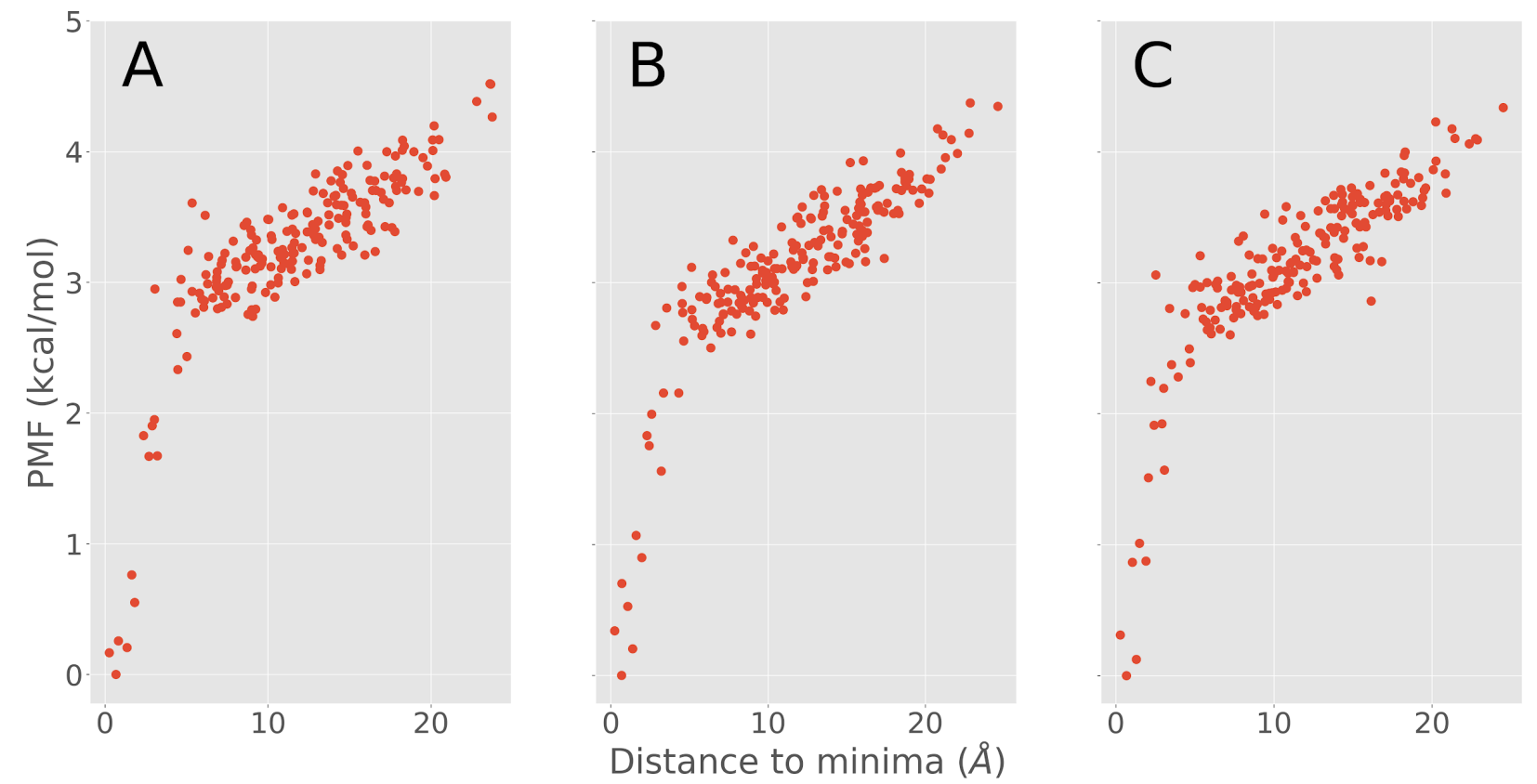

Figure S8. Comparison of the evolution of the one-dimensional PMF with three iterations for the 4UP ligand of the URO receptor
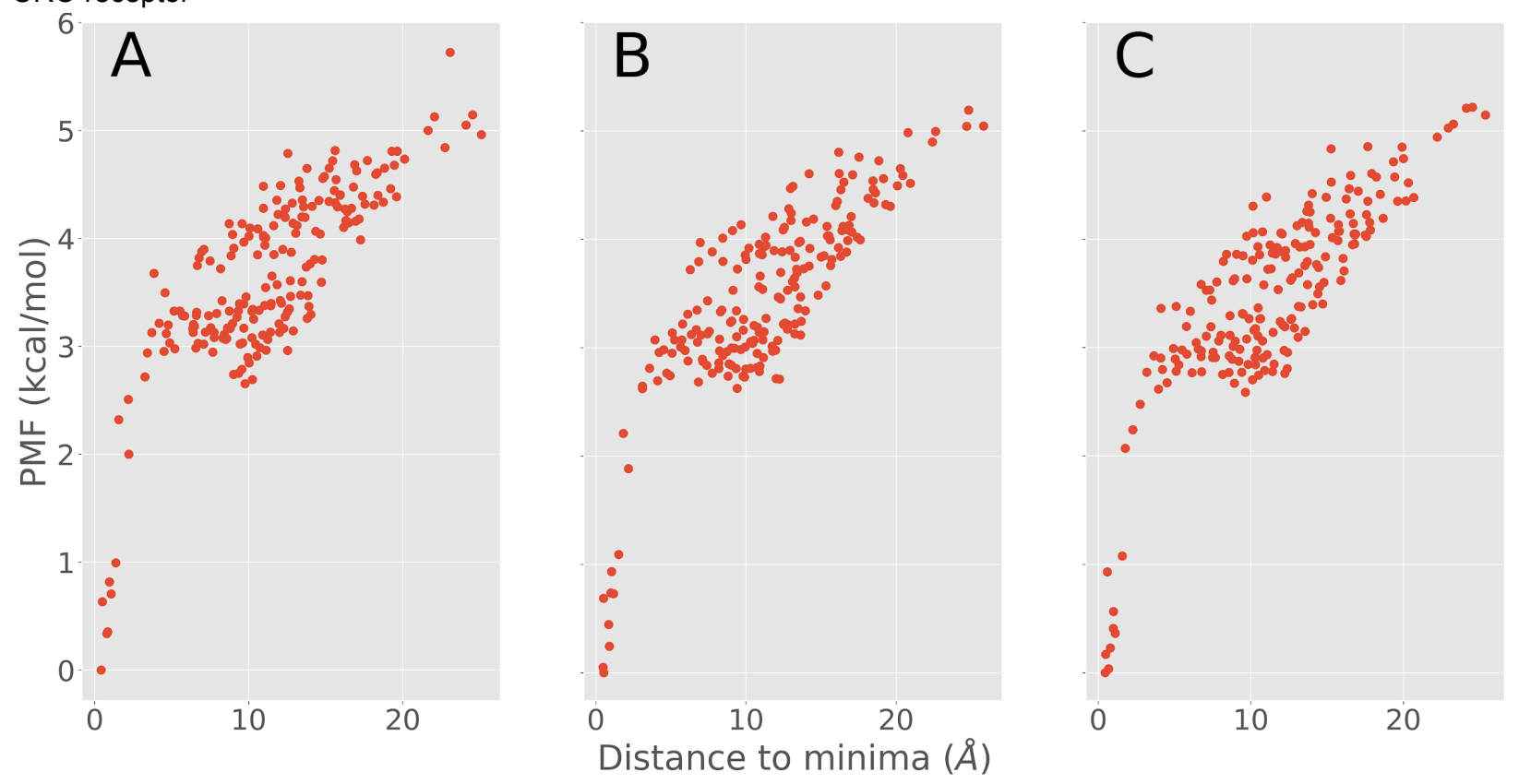

Figure S9. Comparison of the evolution of the one-dimensional PMF with three iterations for the 6UP ligand of the URO receptor 

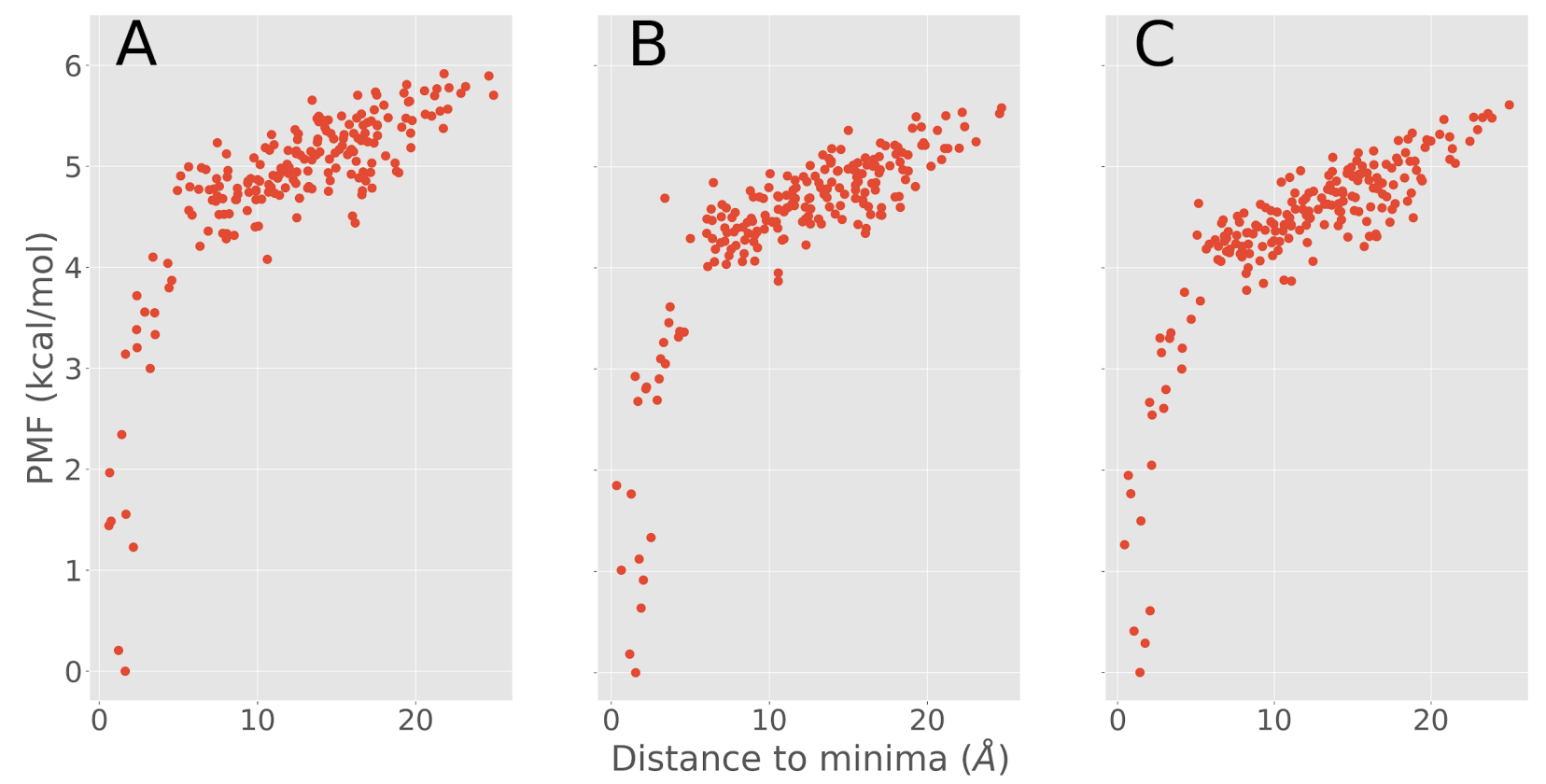

Figure S10. Comparison of the evolution of the one-dimensional PMF with three iterations for the 239 ligand of the URO receptor
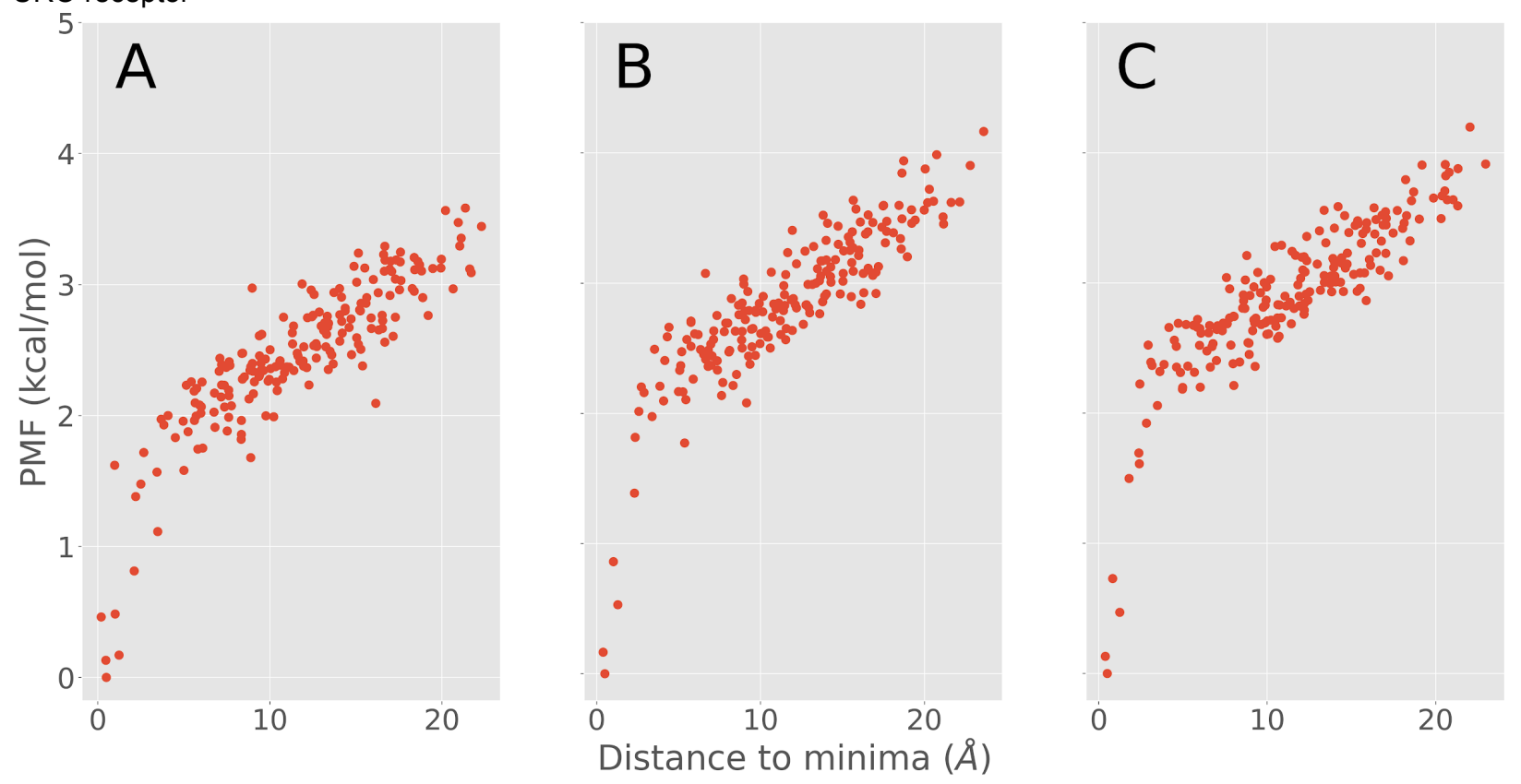

Figure S11. Comparison of the evolution of the one-dimensional PMF with three iterations for the 675 ligand of the URO receptor 

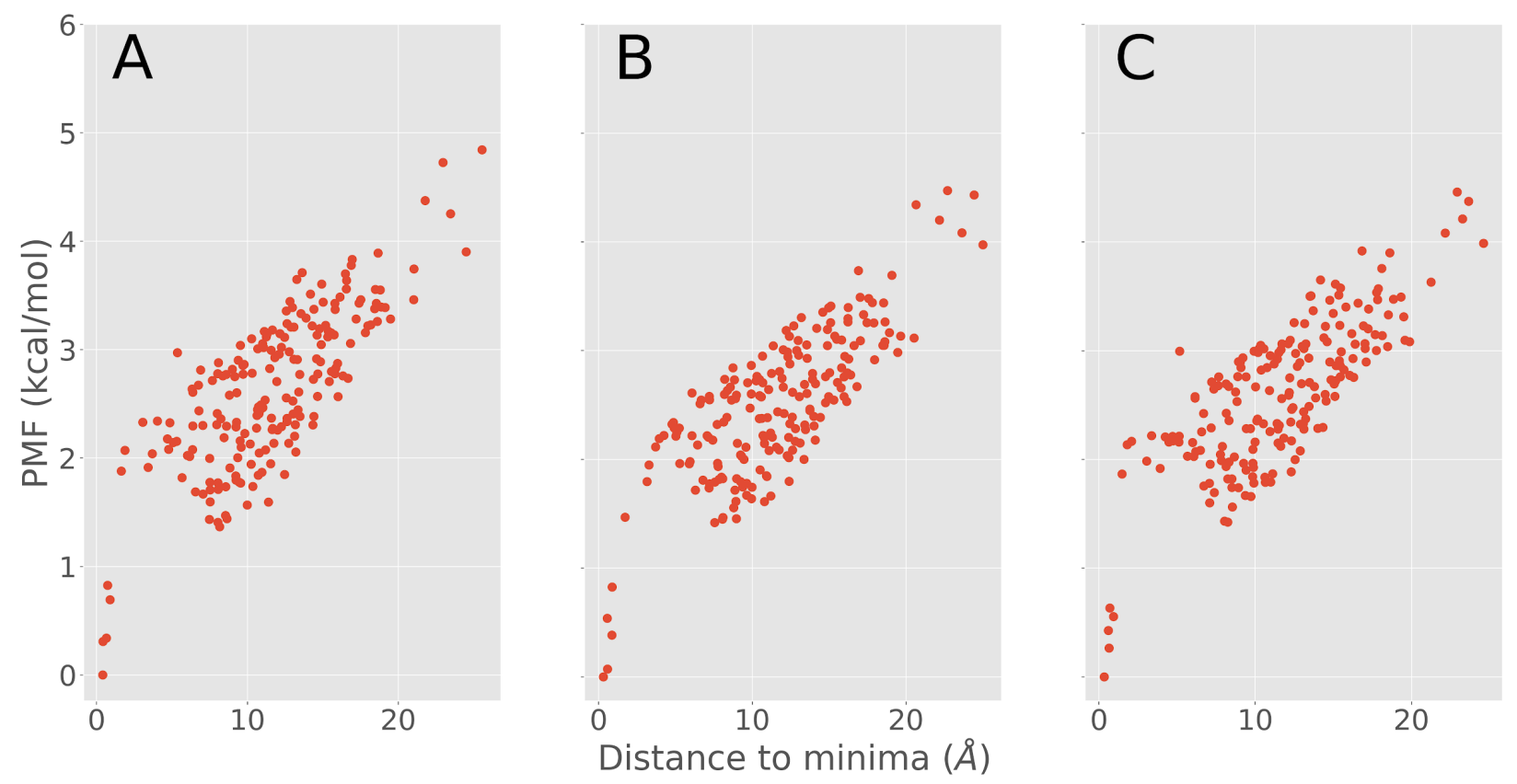

Figure S12. Comparison of the evolution of the one-dimensional PMF with three iterations for the 1UP ligand of the URO receptor

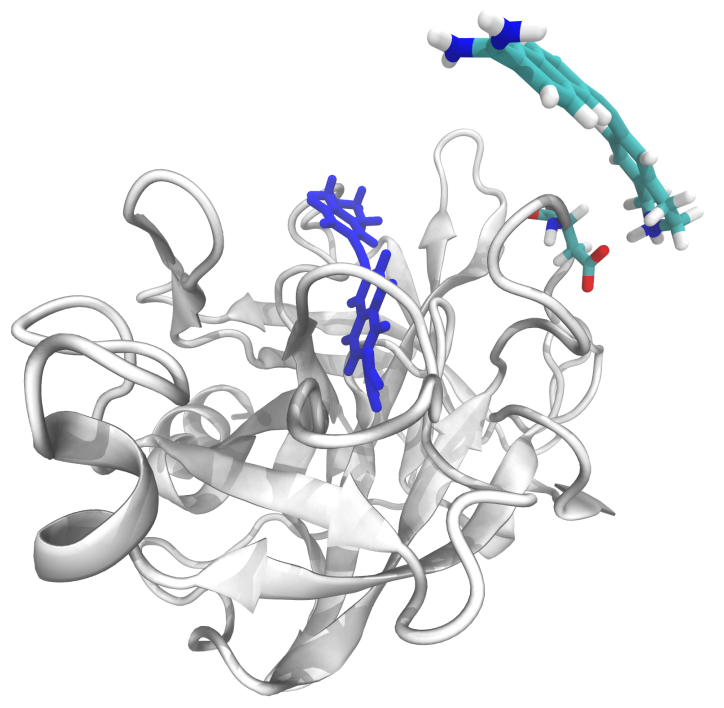

Figure S13. Representative structure of the secondary minimum of the 7UP-URO system 


\section{Results for ERK2 system}

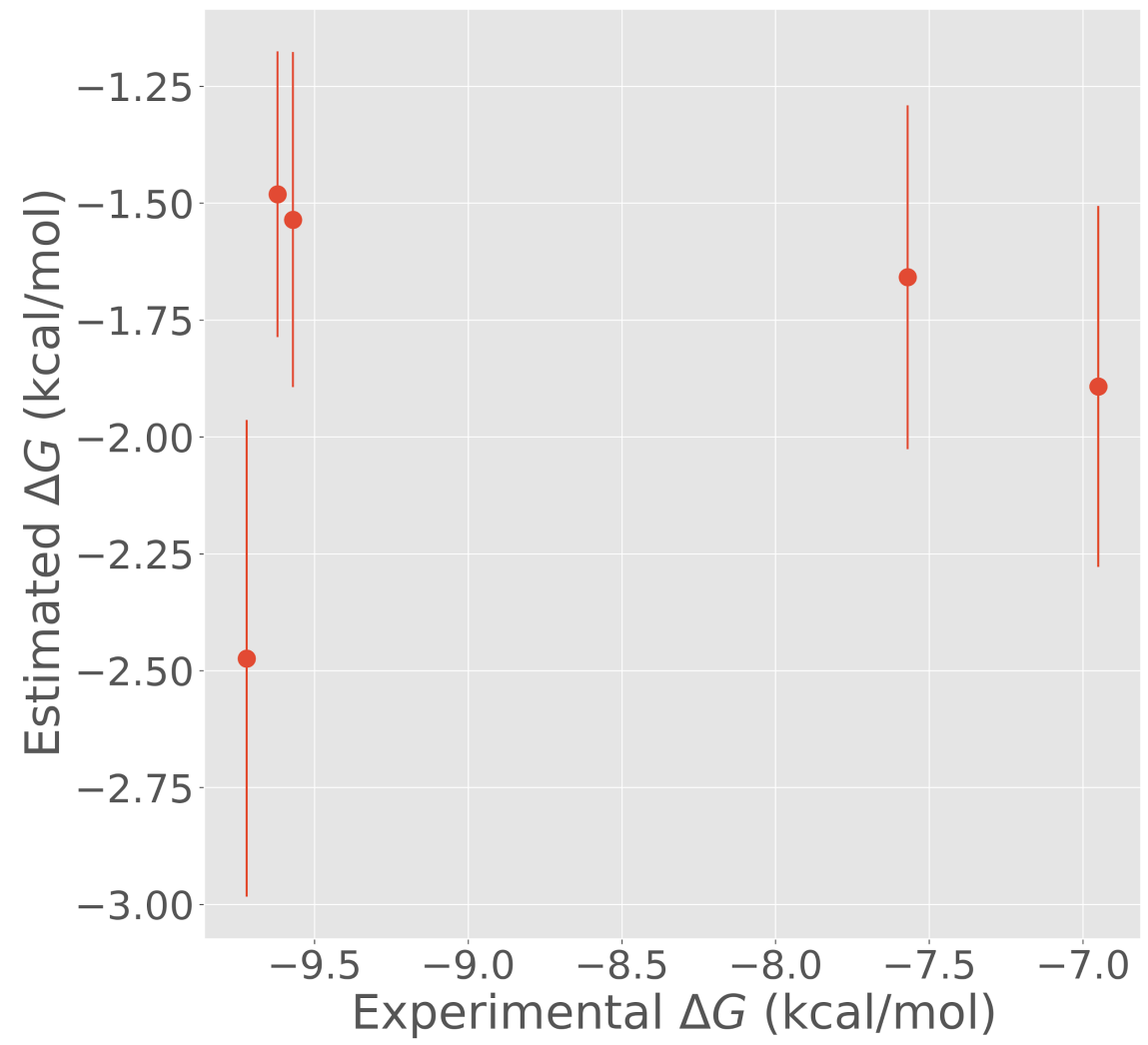

Figure S14. Correlation of the predicted binding free energy with respect to experimental values for the ERK2 system
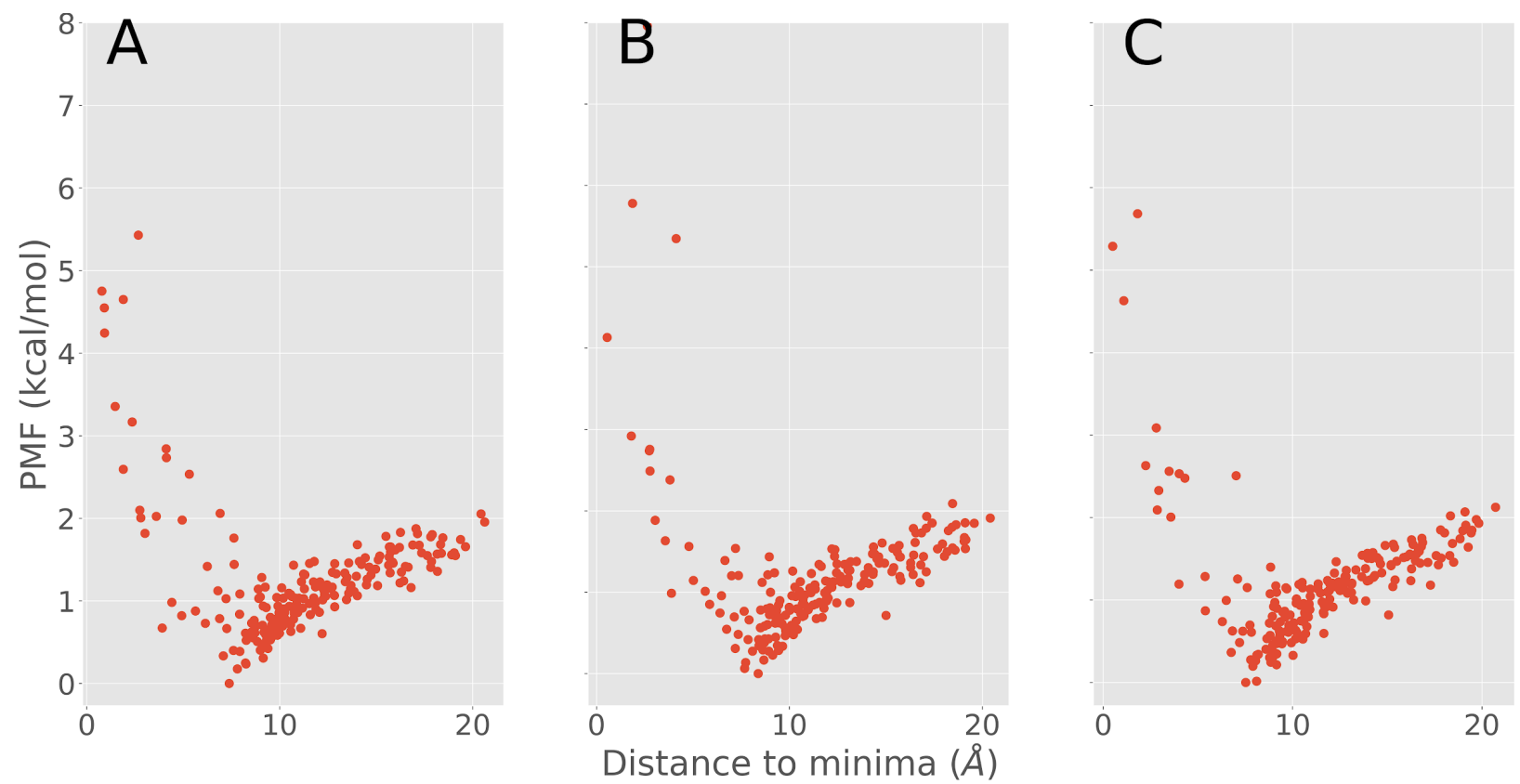

Figure S15. Comparison of the evolution of the one-dimensional PMF with three iterations for the EK3 ligand of the ERK2 receptor 

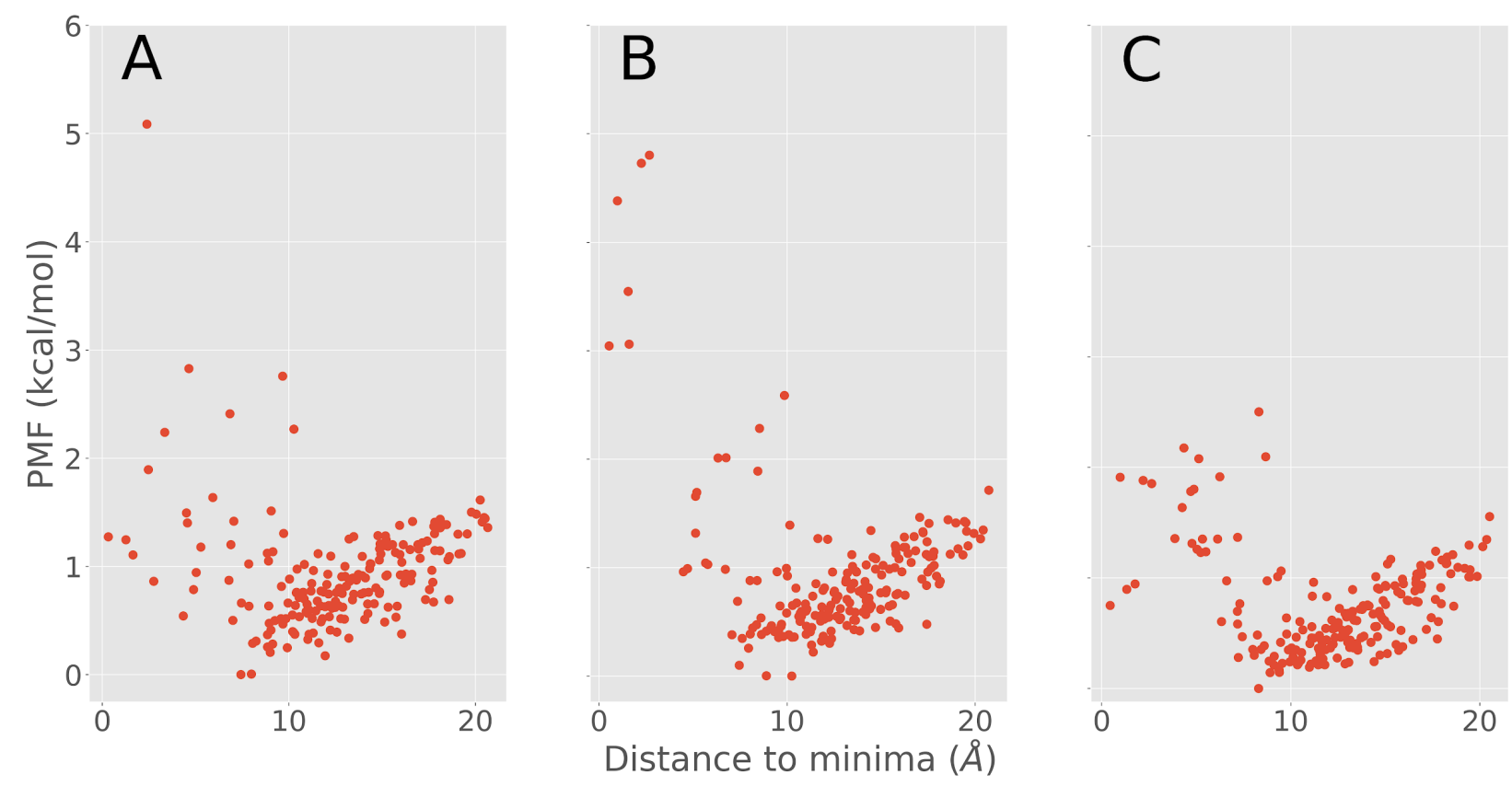

Figure S16. Comparison of the evolution of the one-dimensional PMF with three iterations for the EK6 ligand of the ERK2 receptor
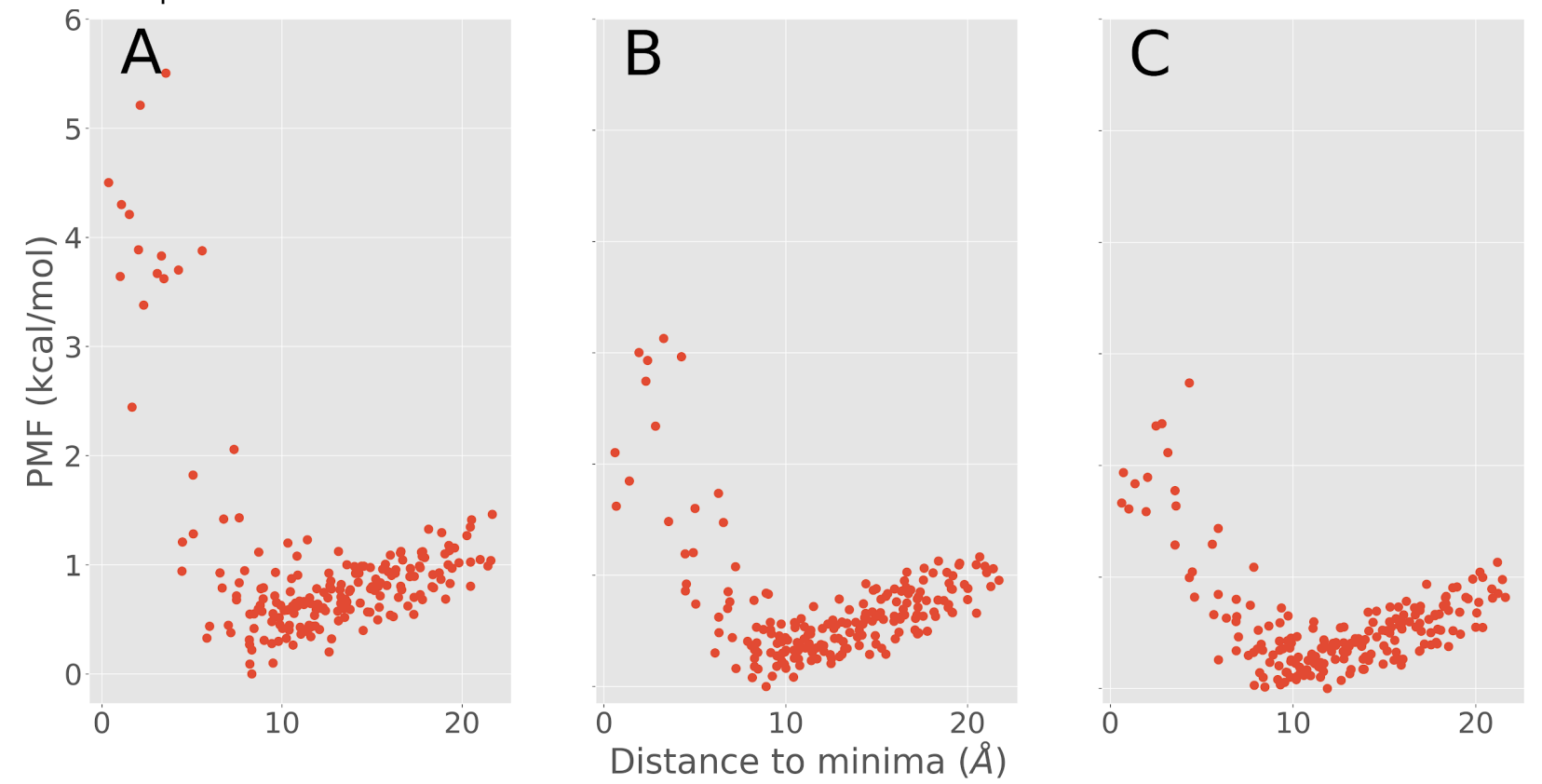

Figure S17. Comparison of the evolution of the one-dimensional PMF with three iterations for the EK9 ligand of the ERK2 receptor 

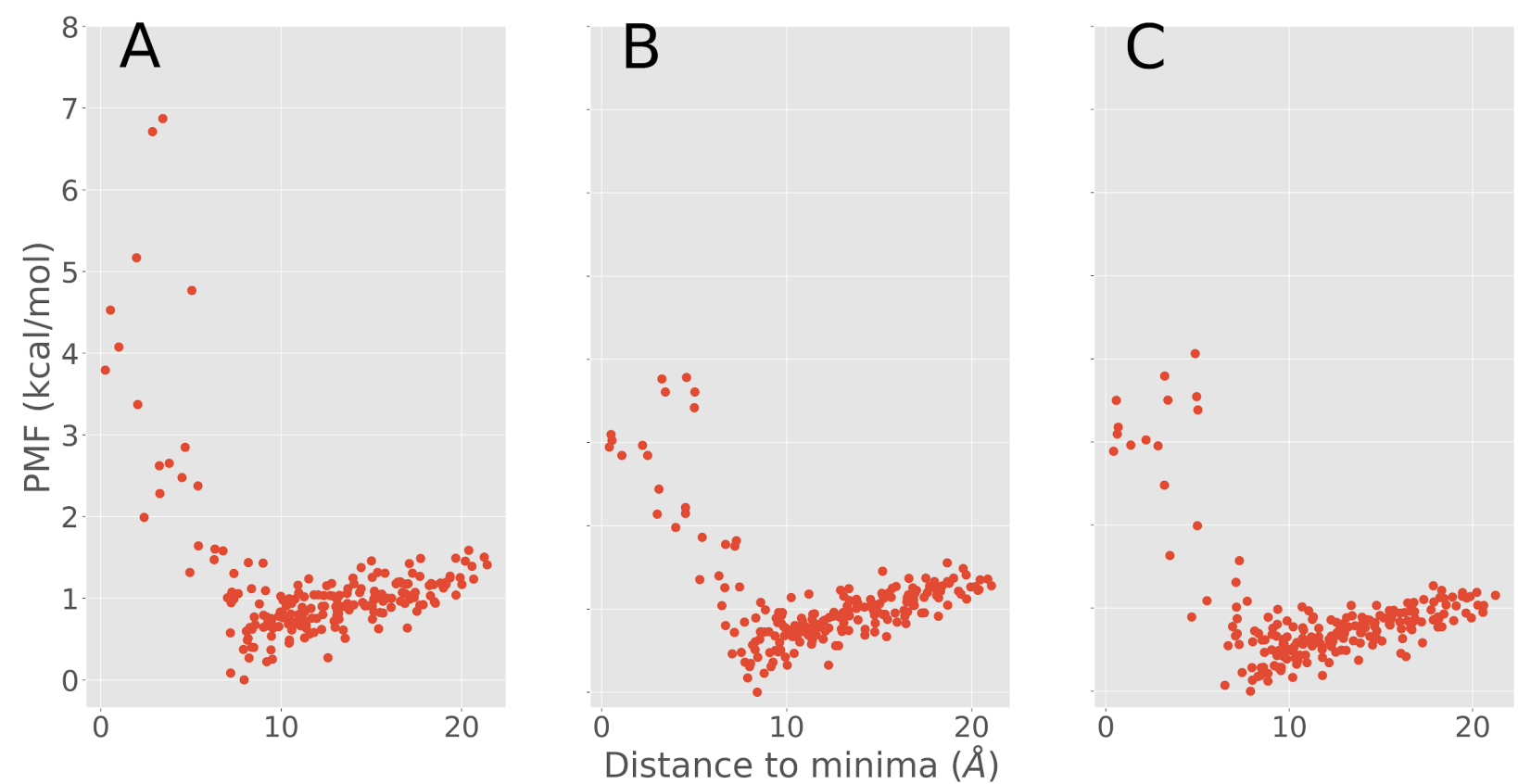

Figure S18. Comparison of the evolution of the one-dimensional PMF with three iterations for the E63 ligand of the ERK2 receptor

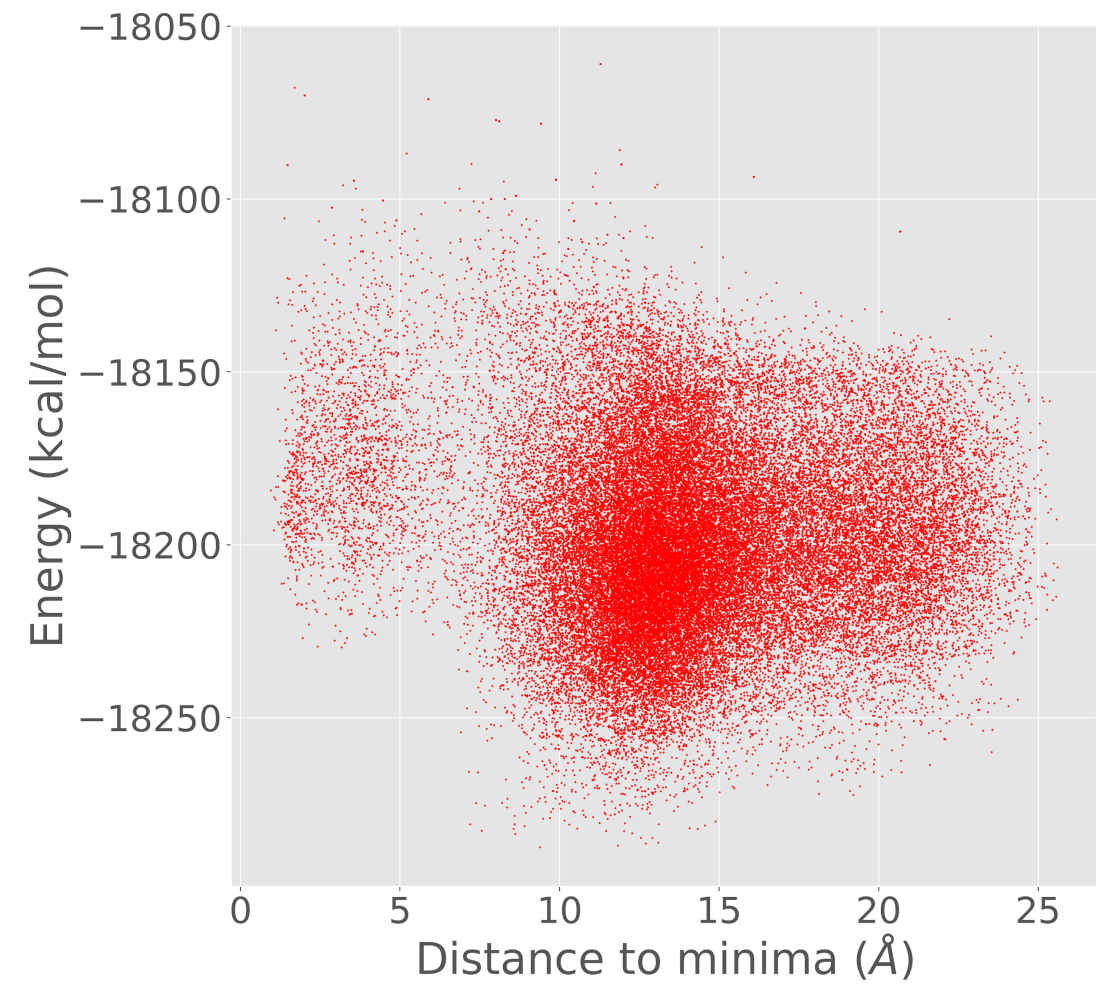

Figure S19. Comparison of the total energy of the three independent simulations with respect to the distance used in the one-dimensional PMF for the EK2 ligand (ERK2 system) 

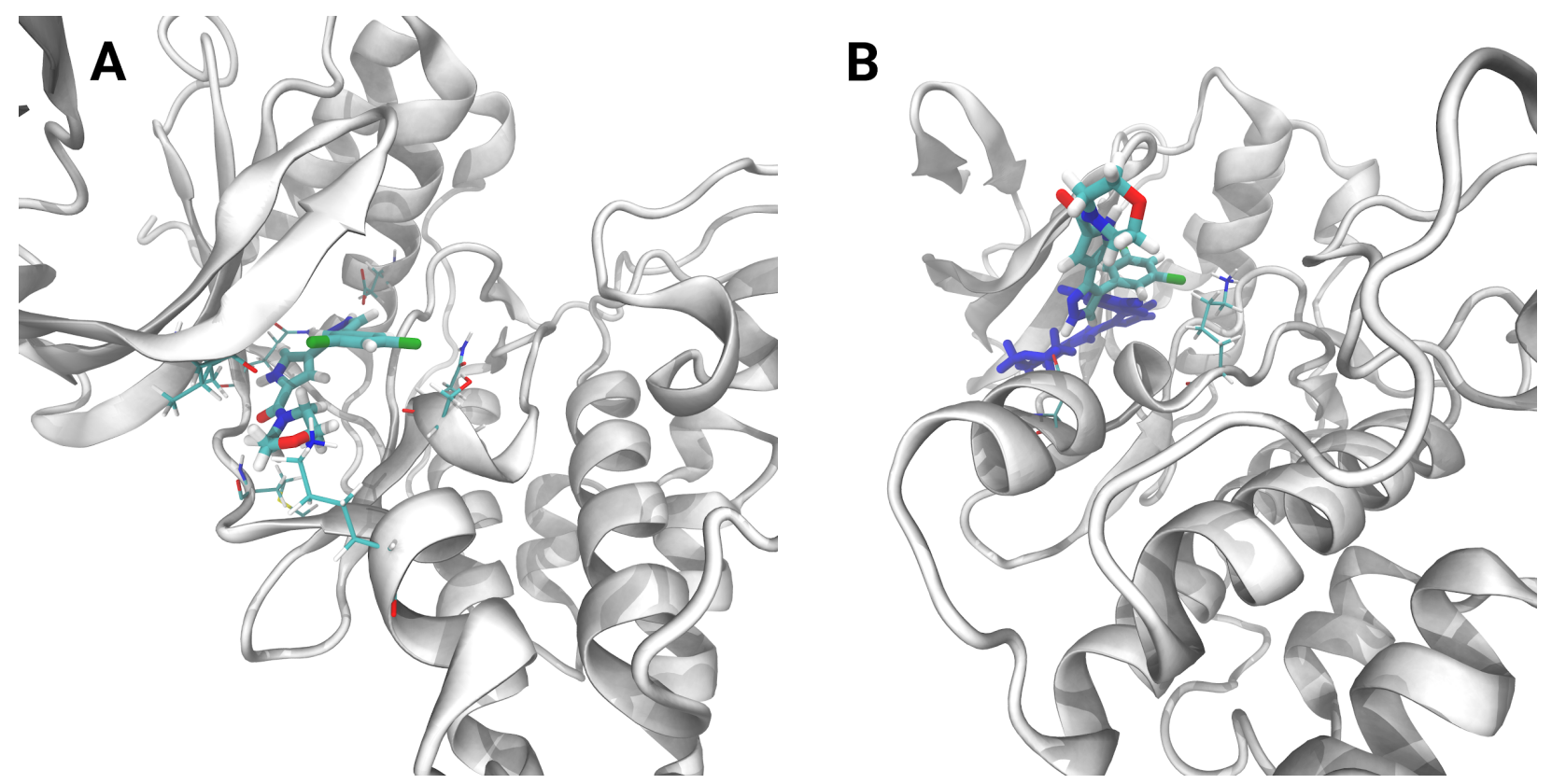

Figure S20. Representative structure of the minima of the EK2 ligand (A) Active site structure (B) Absolute minimum at about $10 \AA$ from the active site, with the active site structure shown in dark blue

\section{Results for PR system}

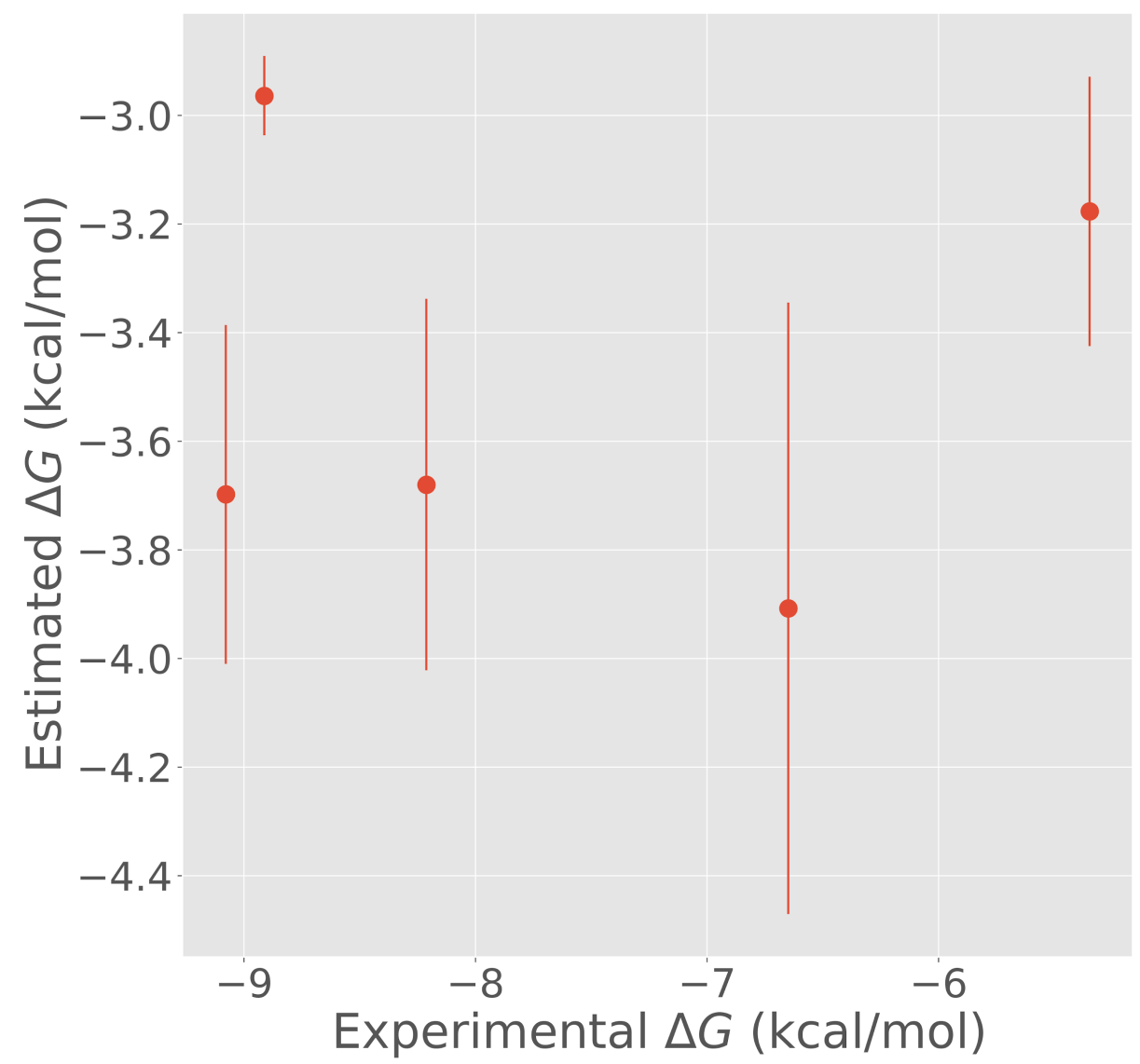

Figure S21. Correlation of the predicted binding free energy with respect to experimental values for the PR system 

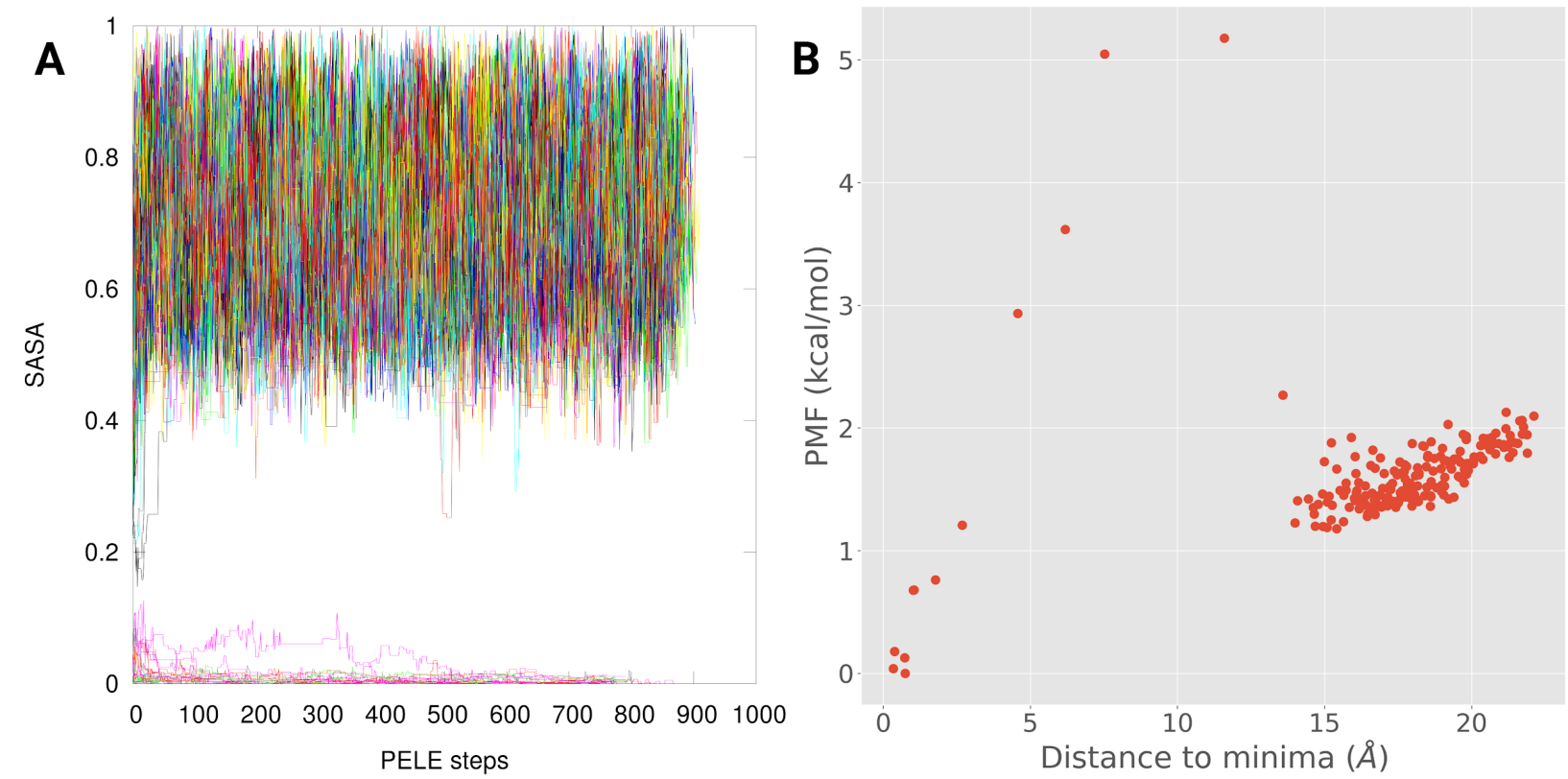

Figure S22. A) Evolution of the SASA for the trajectories from the first iteration of PR with STR ligand (B) PMF of the first iteration

\section{Comparison enhanced sampling use}

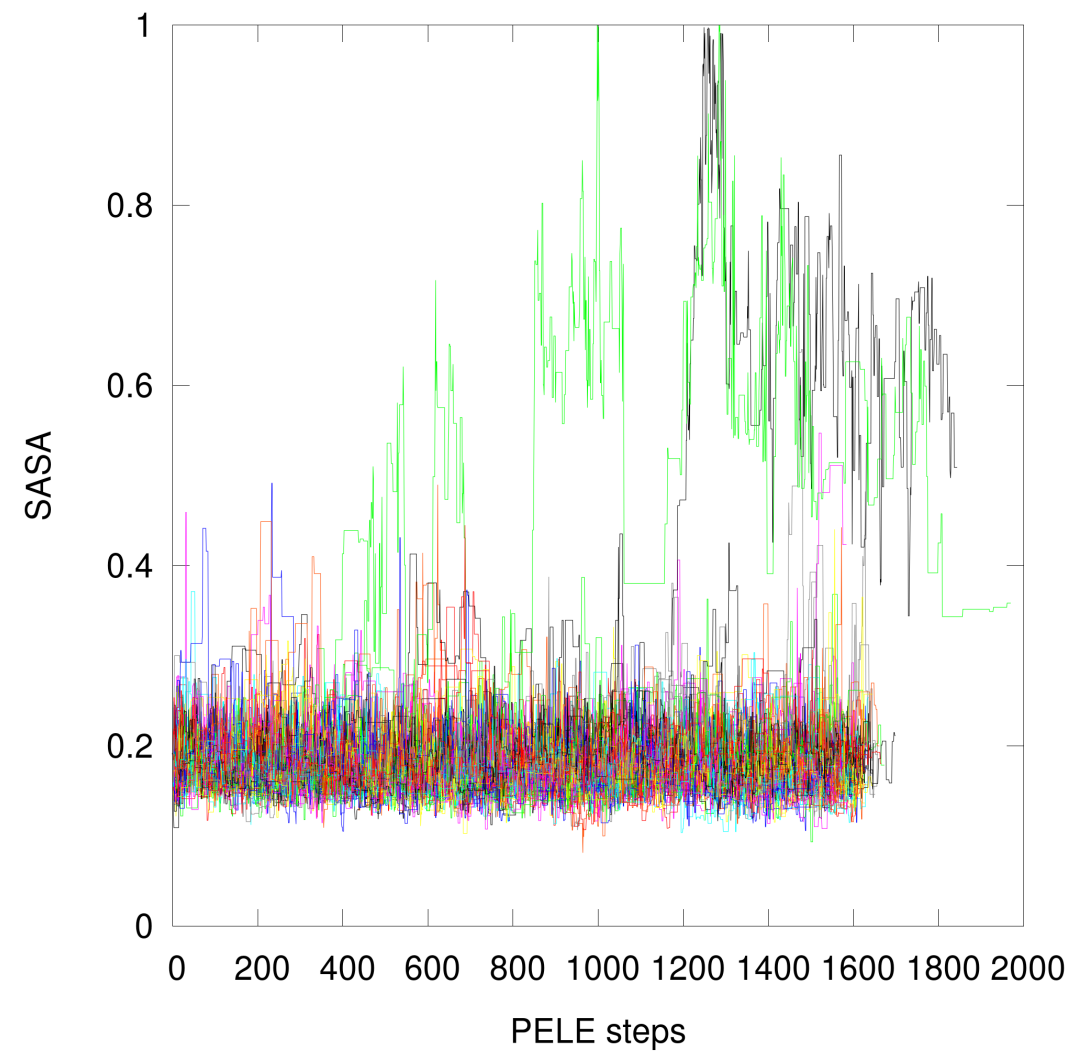

Figure S23. Complete trajectories from the first iteration of the simulation starting from a single bound conformation in the active site for ligand L05 (Plasmin system). 

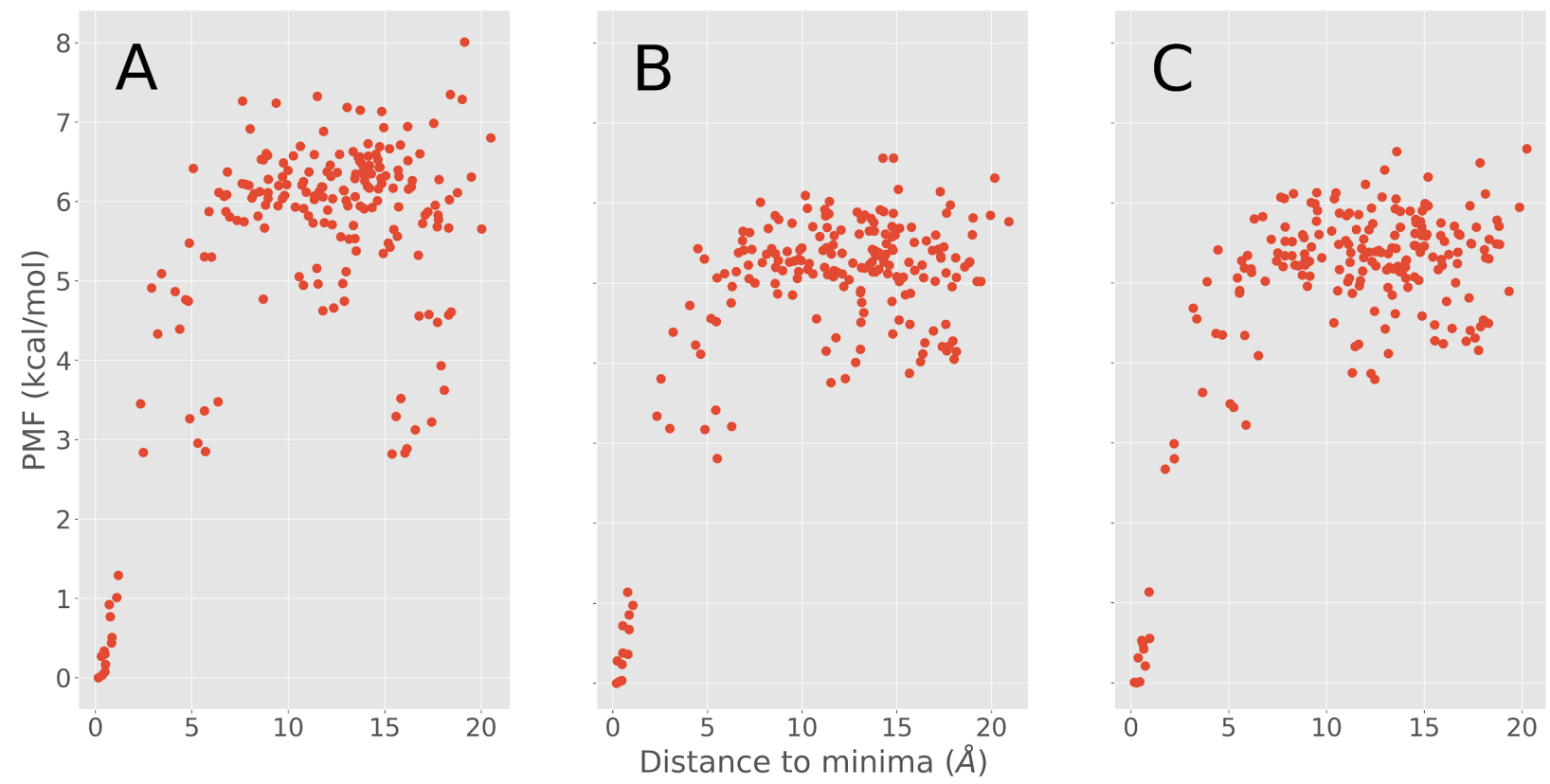

Figure S24. Comparison of the evolution of the one-dimensional PMF with three iterations for the L01 ligand of the Plasmin receptor with a larger solvent box
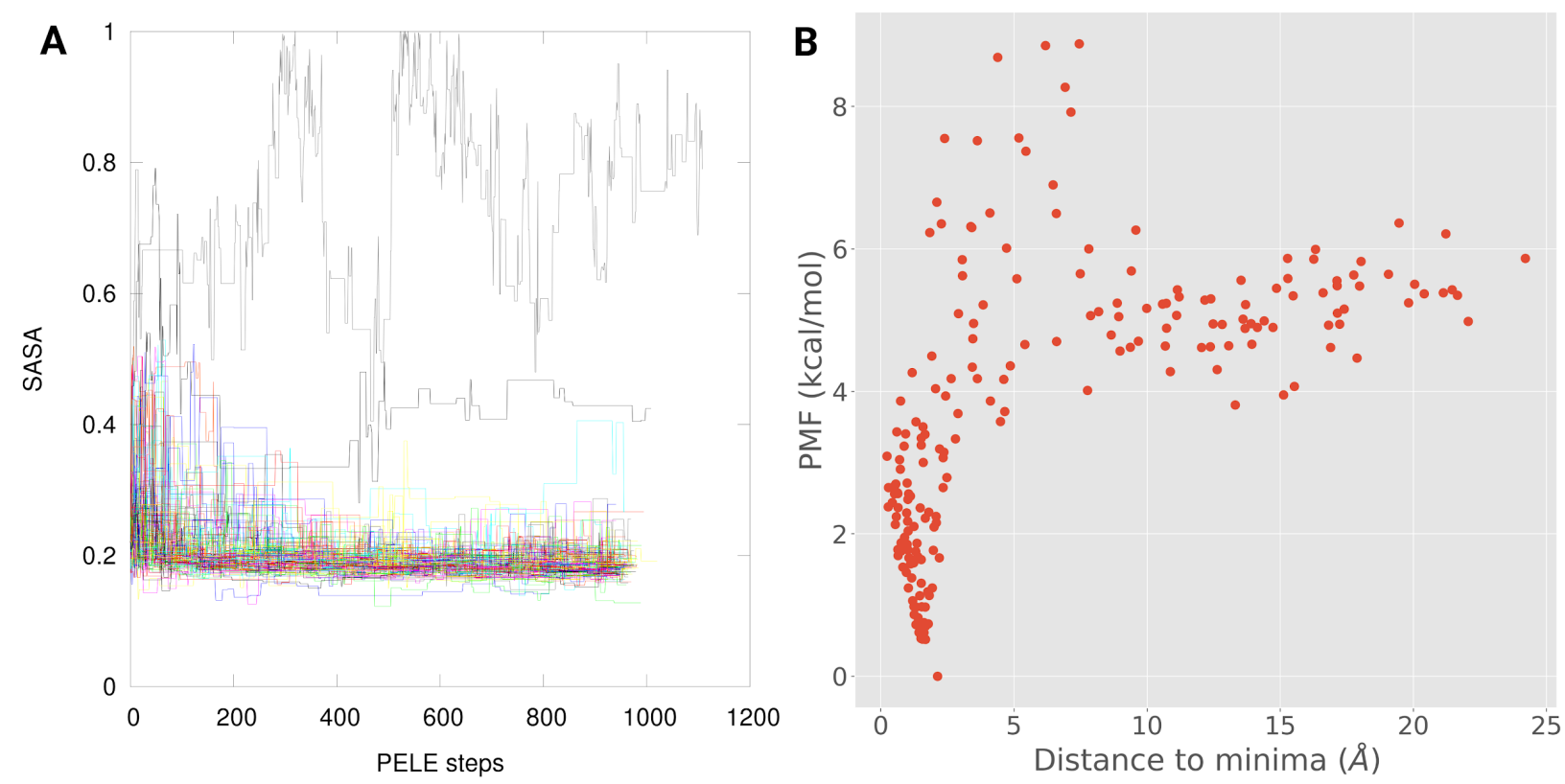

Figure S25. A) Complete trajectories from the first iteration of the simulation starting from a single bound conformation in the active site for ligand 239 (URO system) (B) PMF of the first iteration of the simulation starting from a single conformation in the active site 


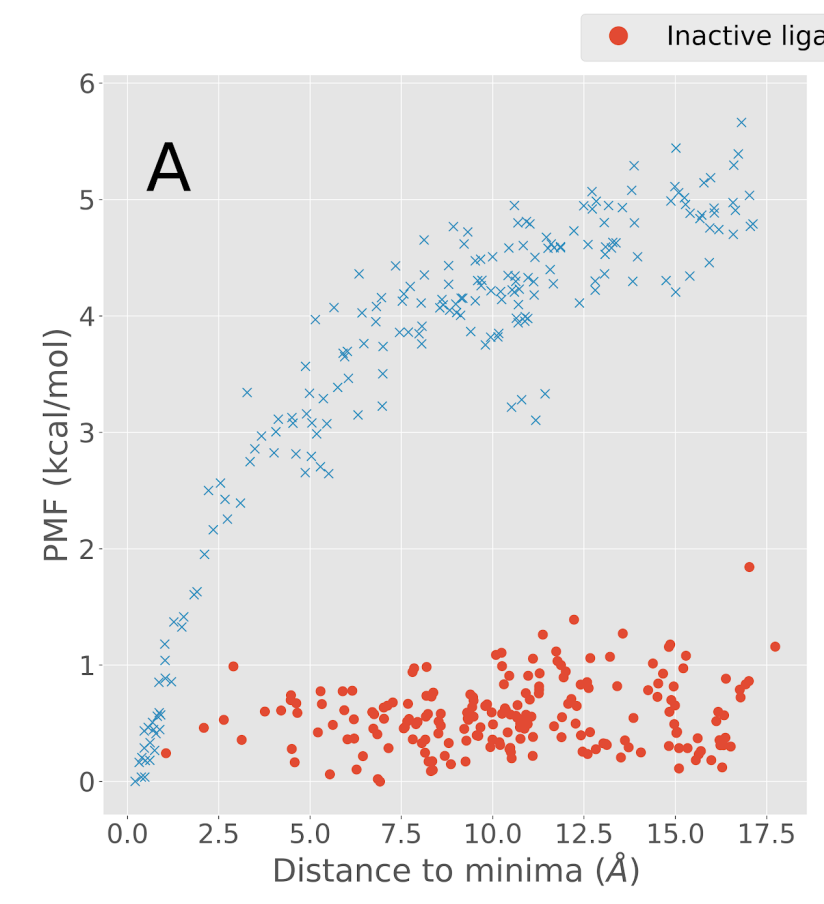

Active ligand

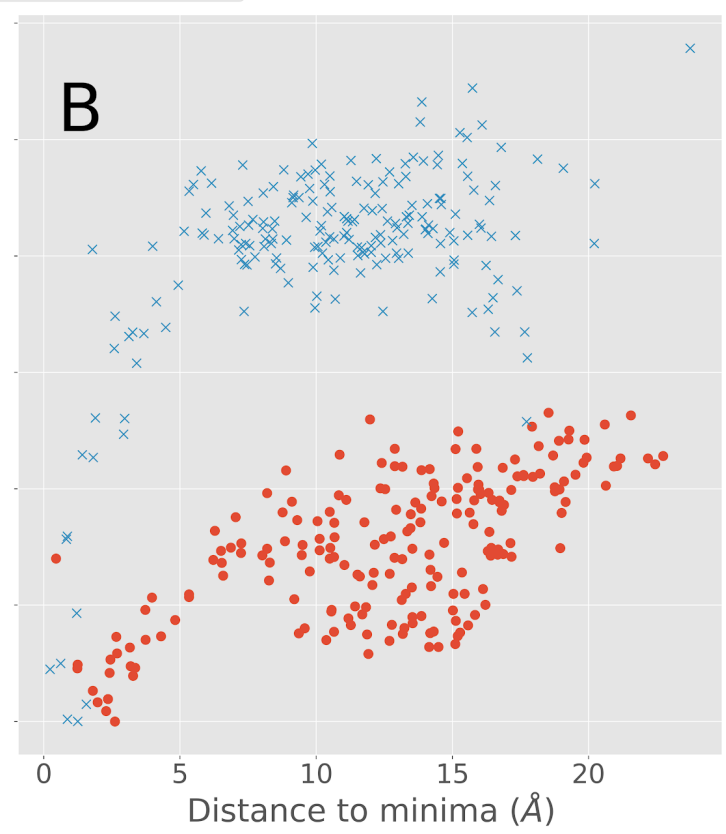

Figure S26. Comparison of the one-dimensional PMF of the inactive modification of the L01 ligand of the Plasmin receptor with respect to $A$ ) the original L01 ligand in the Plasmin receptor and B) the 7UP ligand in the URO receptor. 


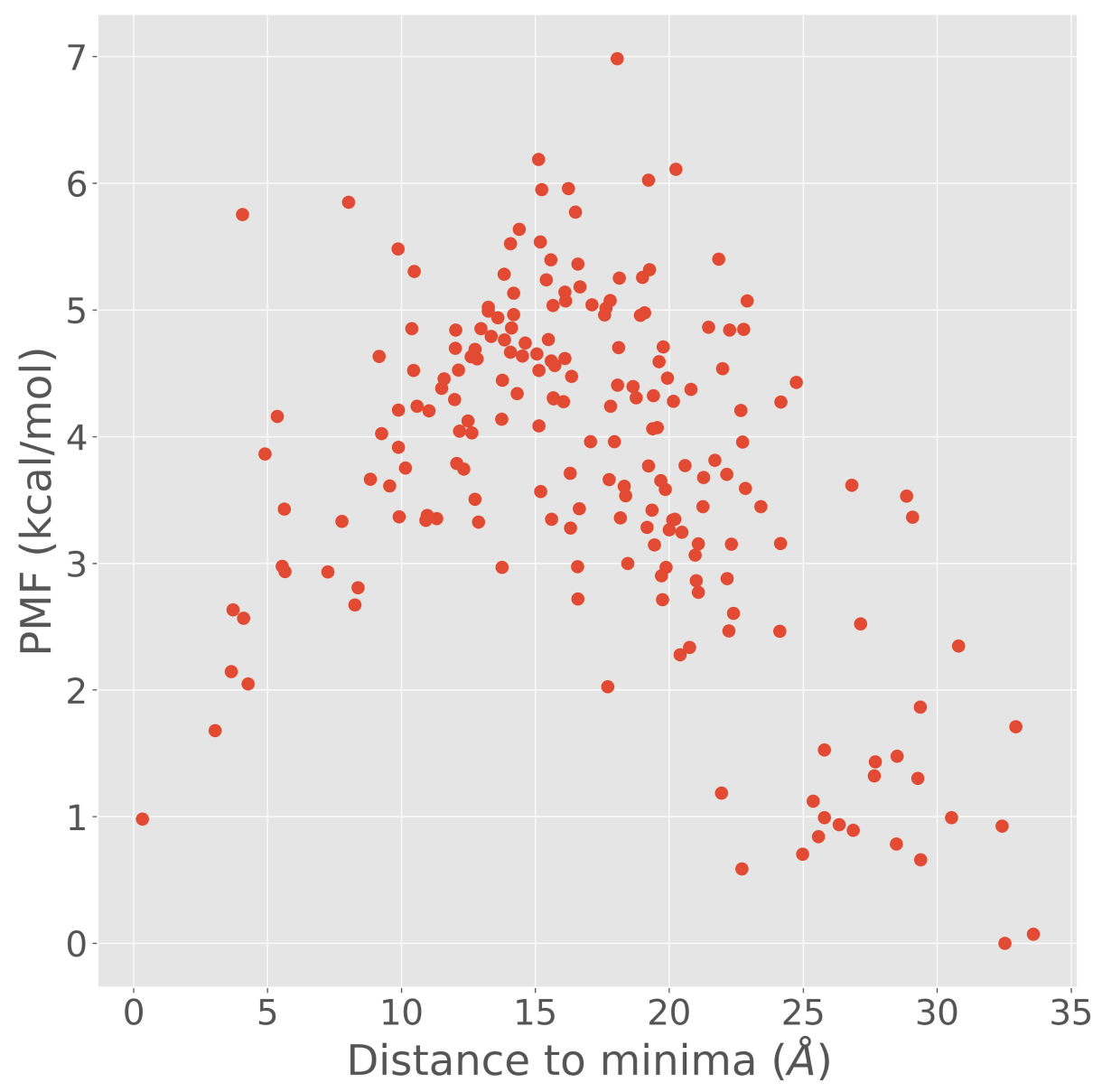

Figure S27. One-dimensional projection of the PMF of the EK2 ligand with the ERK2 receptor introducing four crystallographic waters 